\title{
Prognostic Impact of Genetic Variants of MECP2 and TIRAP on Clinical Outcomes of Systemic Lupus Erythematosus with and without Nephritis
}

\author{
Safaa I. Tayel 1,2,*iD, Nashwa M. Muharram ${ }^{1,2}$, Dina S. Fotoh ${ }^{3}$, Hany S. Elbarbary ${ }^{4,5}$, Huda I. Abd-Elhafiz ${ }^{6}$, \\ Eman A. El-Masry ${ }^{7,8}$, Ahmed E. Taha ${ }^{7,9}$ and Shimaa E. Soliman ${ }^{1,10}$
}

1 Medical Biochemistry and Molecular Biology Department, Faculty of Medicine, Menoufia University, Shebin el Kom 32511, Egypt; nashwa.mouharam@med.menofia.edu.eg (N.M.M.);

Shaimaa_alshafaay@med.menofia.edu.eg (S.E.S.)

2 Medical Biochemistry Unit, College of Medicine, Al Baha University, Al Baha 65779, Saudi Arabia

3 Physical Medicine, Rheumatology and Rehabilitation Department, Faculty of Medicine, Menoufia University, Shebin el Kom 32511, Egypt; dina.salem.12@med.menofia.edu.eg

4 Renal Unit, Department of Internal Medicine, Faculty of Medicine, Menoufia University, Shebin el Kom 32511, Egypt; hany.elbarbari@med.menofia.edu.eg

5 Renal Unit, Department of Internal Medicine, College of Medicine, King Faisal University, Al-Ahsa 31982, Saudi Arabia

6 Clinical Pharmacology Department, Faculty of Medicine, Menoufia University, Shebin el Kom 32511, Egypt; Hoda.break@med.menofia.edu.eg

check for updates

Citation: Tayel, S.I.; Muharram, N.M.; Fotoh, D.S.; Elbarbary, H.S.; Abd-Elhafiz, H.I.; El-Masry, E.A.; Taha, A.E.; Soliman, S.E. Prognostic Impact of Genetic Variants of MECP2 and TIRAP on Clinical Outcomes of Systemic Lupus Erythematosus with and without Nephritis. Biomolecules 2021, 11, 1378. https://doi.org/ 10.3390/biom11091378

Academic Editor: Undurti N. Das

Received: 24 August 2021

Accepted: 12 September 2021

Published: 18 September 2021

Publisher's Note: MDPI stays neutral with regard to jurisdictional claims in published maps and institutional affiliations.

Copyright: (c) 2021 by the authors. Licensee MDPI, Basel, Switzerland. This article is an open access article distributed under the terms and conditions of the Creative Commons Attribution (CC BY) license (https:/ / creativecommons.org/licenses/by/ $4.0 /)$.
7 Microbiology and Immunology Unit, Department of Pathology, College of Medicine, Jouf University, Sakaka 72388, Saudi Arabia; ealmasry@ju.edu.sa (E.A.E.-M.); aeattia@ju.edu.sa (A.E.T.)

8 Medical Microbiology and Immunology Department, Faculty of Medicine, Menoufia University, Shebin el Kom 32511, Egypt

9 Medical Microbiology and Immunology Department, Faculty of Medicine, Mansoura University, Mansoura 35516, Egypt

10 Medical Biochemistry Unit, Department of Pathology, College of Medicine, Qassim University, Buraydah 51452, Saudi Arabia

* Correspondence: drsafaa_tayel@yahoo.com or safaa.tail@med.menofia.edu.eg; Tel.: +20-10-033-83097

Abstract: Systemic lupus erythematosus (SLE) is a chronic autoimmune illness with a growing prevalence in many populations. Few studies have examined genetic predisposition to SLE, so we aimed to examine the clinical impact of the genetic polymorphisms MECP2 rs2734647and TIRAP rs8177374 on the outcomes and therapeutic precision of SLE with and without nephritis. This study included 110 SLE patients—divided into 63 with lupus nephritis (LN), and 47 without nephritis—and 100 controls. Laboratory measurements including CRP, ESR, ACR, CBC, anti-ds-DNA, vitamin A, C3, and $C 4$ were carried out, along with genotyping of MECP2 rs2734647and TIRAP rs 8177374 by realtime PCR and sequencing. Treg \%, vitamin A, C3, and C4 were lower, whereas Th17 \% was higher, in patients vs. controls $(p<0.001)$. The T allele of MECP2 rs2734647 was higher in LN than in nonnephritis and control subjects. Moreover, the T allele of TIRAP rs8177374 was higher in LN than in non-nephritis and control subjects. The MECP2 and TIRAP genes could play a role in predisposition to SLE, and can also predict disease progress to nephritis, helping to personalize medicine.

Keywords: arthritis; MECP; nephritis; systemic lupus erythematosus; TIRAP

\section{Introduction}

Systemic lupus erythematosus (SLE) is a chronic autoimmune disease that causes the deposition of immune complexes and inflammatory cells in various bodily tissues, including the kidneys. Lupus nephritis (LN) is seen frequently in SLE, and is one of the most serious SLE complications, since it is the main forecaster of poor prognosis. The prevalence of LN differs depending on the studied population, being more common in people with Asian (55\%), African (51\%), and Hispanic (43\%) ancestry compared with 
Caucasians (14\%). Approximately $25 \%$ of LN patients still develop end-stage renal disease (ESRD) 10 years after the onset of renal affection [1,2].

Toll-like receptors (TLRs) are crucial originators of the immune response, both innate and acquired. Gene polymorphisms within TLRs trigger faults in vital TLR-related signaling routes, which subsequently raise the risk of autoimmune diseases [3].

The Toll-interleukin-1 receptor (TIR) domain-containing adaptor protein (TIRAP) signifies a fundamental intracellular signaling molecule controlling various immune responses. TIRAP is an adaptor protein that pairs myeloid differentiation factor 88 (MyD88) with TLRs to stimulate MyD88-dependent TLR signaling. The subsequent downstream signaling processes end in the triggering of diverse transcription factors including nuclear factor $\mathrm{KB}(\mathrm{NF}-\mathrm{KB})$ and activated protein 1 (AP1). The instigation of TLR-mediated signaling pathways is crucial in the induction of proinflammatory cytokines by immune cells, and in monitoring host cell survival $[4,5]$.

SNPs in the TIRAP gene have been linked to the initiation of, and susceptibility to, inflammatory diseases such as SLE [6]. TIRAP rs8177374 (C/T) SNP, which causes a leucine substitution at serine 180 of Mal (S180L), is associated with an increase in susceptibility to infectious diseases such as malaria, tuberculosis, and septic shock; moreover, S180L leads to an amino acid substitution in which Mal alters TLR2 and TLR4 signaling; hence, it could protect against life-threatening inflammatory disorders [7].

Improvements in immunosuppressive drugs and other pharmacological treatments have increased the mean 5-year survival for SLE patients. However, the lack of specificity, wide suppression of the immune cell functions, and effects on the replication of those cells frequently lead to serious toxicity and numerous adverse effects, so understanding the genetic polymorphism of different patients may provide a new insight into the molecular pathogenesis of LN and aid in the development of novel diagnostic and therapeutic tools [8].

Epigenetic mechanisms are heritable events that control gene expression by regulating the accessibility of DNA to the transcriptional complex [9]. Alterations of those mechanismsincluding DNA methylation, histone modifications, and non-coding transcripts-are involved in the deregulation reported in many autoimmune/inflammatory diseases, including SLE [10].

One important gene, which contains the risk variant for autoimmune diseases as SLE according to numerous studies, is Methyl-CpG-binding protein 2 (MECP2)-a transcriptional regulator that controls the expression of methylation-sensitive genes. MECP2 recruits DNA methyltransferase 1 (DNMT1) during DNA methylation; it also recruits histone deacetylase complexes to the gene promoter, leading to chromatin conformation and, hence, silencing of gene expression [11].

Few studies have demonstrated the influence of methylation status on TLR expression in various illnesses, although one report declared that hypomethylation at gene promoters of TLR1, 2, 4, 6, 8, and 9 augments the expression of downstream genes, and treating Kawasaki disease patients with intravenous immunoglobulin can reduce TLRs' mRNA expression and establish their methylation. Meanwhile, another report showed that CNS inflammation may be dysregulated by different means, where the TLR3, TLR9, RAGE, and MECP2 proteins could contribute [12,13].

Altered regulation of T-cell genes, which are sensitive to methylation, along with the fact that SLE is more common in women, makes the MECP2 gene an important genetic factor of SLE [14]. The current study aimed to investigate the clinical impact of the genetic polymorphisms MECP2 rs2734647and TIRAP rs8177374 on the clinical outcomes and selective management of SLE with or without nephritis.

\section{Materials and Methods}

This hospital-based case-control study was conducted on 110 Egyptian SLE patients recruited from the outpatient clinic of the Rheumatology, Physical Medicine, and Rehabilitation department, and the Nephrology clinic of the Internal Medicine Department, 
Menoufia University, between January and December 2020, in collaboration with the Medical Biochemistry and Molecular Biology, Medical Microbiology and Immunology, and Clinical Pharmacology departments. We classified 110 SLE patients based on evidence of clinical and laboratory characteristics of nephritis into two subgroups: 63 patients with LN, and 47 patients without nephritis. Moreover, 100 Egyptian healthy age- and sex-matched controls were also enrolled in the study. All patients and control subjects were females. The diagnosis of SLE was made as per the American College of Rheumatology (ACR) and the European League Against Rheumatism (EULAR)'s 2019 criteria for the classification of SLE $[15,16]$. The ACR/EULAR classification requires an antinuclear antibody (ANA) titer of at least 1:80 in HEp-2 cells, or an equivalent positive test, at least once. If that is present, 22 "additive-weighted" classification criteria are considered, comprising 7 clinical domains (constitutional, hematologic, neuropsychiatric, mucocutaneous, serosal, musculoskeletal, and renal) and 3 immunological domains (antiphospholipid antibodies, complement proteins, and SLE-specific antibodies). Each criterion is assigned a number of points, ranging from 2 to 10. Patients with at least one clinical criterion and 10 or more points are classified as having SLE. Laboratory tests indicated for SLE diagnosis include CBC with differential, serum creatinine, urinalysis with microscopy, ESR, CRP level, complement levels, liver function tests, spot protein/spot creatinine ratio, and autoantibody tests. Active disease is characterized by a systemic lupus erythematosus disease activity index (SLEDAI) score of more than 3 [17]. Laboratory tests for SLE disease activity include the following: antibodies to double-stranded DNA (dsDNA), complement $(\mathrm{C} 3, \mathrm{C} 4)$ levels, erythrocyte sedimentation rate (ESR), and C-reactive protein (CRP).

Kidney biopsy was used to confirm the presence of LN, to aid in the classification of systemic lupus erythematosus (SLE) nephritis based on the International Society of Nephrology/Renal Pathology Society (ISN/RPS) classification, and to guide therapeutic decisions in the presence of the following features [18]: increased serum creatinine in the absence of strong evidence for another etiology; proteinuria of more than $1.0 \mathrm{~g}$ per $24 \mathrm{~h}$, as confirmed by $24 \mathrm{~h}$ urine specimens or spot protein/spot creatinine ratios; proteinuria of $0.5 \mathrm{~g}$ or more per $24 \mathrm{~h}$, along with either hematuria ( $\geq 5 \mathrm{RBCs} / \mathrm{hpf}$ ) or cellular casts, as confirmed by a minimum of 2 tests within a short period. Additionally, in the absence of alternative causes, and during regular follow-up, laboratory abnormalities suggesting active lupus nephritis include hematuria or proteinuria. Laboratory tests to evaluate renal function in SLE patients with renal involvement included the following: blood urea nitrogen (BUN) testing, serum creatinine assessment, urinalysis (to check for protein, red blood cells (RBCs), and cellular casts) and spot urine tests for creatinine and protein concentration, and $24 \mathrm{~h}$ urine tests for creatinine clearance and protein excretion.

Patients with severe infection, immunodeficiency, malignancy, thyroid dysfunction, chronic liver disease, or chronic kidney disease from a different cause were excluded from our study. Male patients were excluded from the study due predominance of the disease in females-SLE is nearly 9 times more common in women than men over the life span [19]. In this regard, SLE appears to be one of the most sex-differentiated autoimmune diseases. Because SLE is most commonly diagnosed at reproductive age, the disease presents medical and psychosocial challenges that complicate family planning and pregnancy $[20,21]$.

This study was conducted in accordance with the ethical standards of our institute and the 1964 Declaration of Helsinki. All participants gave informed consent before taking part in the study. This study was approved by the research ethical committee of the faculty of medicine, Menoufia University.

Full history and complete general and local joint examination were performed, along with pain evaluation using a visual analogue scale (VAS) [22]. Laboratory investigationsincluding complete blood count (CBC) using a Pentra-80 automated blood counter (ABXFranc-Rue du Caducee-Paris Euromedecine-BP-7290.34184 Montpellier-Cedex4), and erythrocyte sedimentation rate (ESR) - were conducted. Renal function tests (serum creatinine and blood urea) were performed via colorimetric methods using DIAMOND diagnostics kits (Germany.) Albumin-creatinine ratio (ACR) and albuminuria were measured 
using a solid-phase enzyme-linked immunosorbent assay (ELISA) kit provided by DRG international Inc. (USA; cat no EIA-2361) from early morning spot urine collections and adjusted for urinary creatinine. Liver enzymes (alanine aminotransferase (ALT) and aspartate aminotransferase (AST)) were assayed via a kinetic UV-optimized IFCC method (LTEC Kit, England); CRP was measured via ELISA using a Sun Red Elisa Kit (China, catalog no. 201-12-1799). Antinuclear antibodies (ANAs) and human anti-double-stranded-DNA antibody (Anti-dsDNA) were measured using the MBS702970 and MBS269122 ELISA kits, respectively. Serum complement levels were measured using the Complement C3 ELISA Kit (ab108823) and Complement C4 ELISA Kit (ab108824). SLEDAI was used to assess the disease activity [18].

All SLE patients were followed up regularly to assess their renal functions and ACR. All SLE patients started their treatment systematically according to their clinical symptoms, usually with $200 \mathrm{mg}$ of chloroquine phosphate twice daily, and corticosteroids for the treatment of skin manifestations and arthralgia. In case of insufficient clinical improvement, we added $50 \mathrm{mg}$ of azathioprine twice per day with CBC follow up. Most patients with LN received $500 \mathrm{mg}$ of mycophenolate mofetil, starting with two capsules per day, and potentially progressing up to six capsules per day after ACR follow-up. In advanced stages of LN, hospitalization, and administration of cyclophosphamide through IV infusion for 6 months was sometimes necessary.

Ten-milliliter blood samples were collected from each individual under aseptic conditions, and then divided into three parts: (a) $5 \mathrm{~mL}$ added to sterile tubes containing EDTA for complete blood count and CD markers, and DNA extraction for genotyping of MECP2 rs2734647and TIRAP rs8177374 by real-time polymerase chain reaction (PCR); (b) $3.4 \mathrm{~mL}$ added to sterile plain tubes for assessment of vitamin A level, C-reactive protein, and serum creatinine; (c) $1.6 \mathrm{~mL}$ of blood delivered into a tube containing $0.4 \mathrm{~mL}$ trisodium citrate for erythrocyte sedimentation rate.

For genotyping of the MECP2 rs2734647and TIRAP rs 8177374 polymorphisms, DNA extractions were performed from peripheral blood utilizing a Gene JET Whole Blood Genomic DNA Purification Mini Extraction Kit (Thermo Scientific, EU/Lithuania). Genotyping of the C/T polymorphisms within the MECP2 rs2734647 and TIRAP rs8177374 genes was carried out via real-time PCR and allelic discrimination assay utilizing a TaqMan probe (Applied Biosystems, USA). The primers, probes, and Master Mix $(80 \times)$ were also supplied by Thermo Scientific. The used probe sequences for the MECP2 rs2734647 gene were [VIC/FAM]:GTGGCGTTTCTGGGTGTCCCCTGTG [C/T] CTTTTGATATGGGAATACAGCATCA. The used probe sequences for the TIRAP rs8177374 gene were [VIC/FAM]: GAGGGCTGCACCATCCCCCTGCTGT[C/T] GGGCCTCAGCAGAGCTGCCTACCCA. Next, $1.5 \mu \mathrm{L}$ of the primer/probe mixture was applied to a mixture of $10 \mu \mathrm{L}$ of Master Mix and $3.5 \mu \mathrm{L}$ of nuclease-free water. Five microliters of extracted DNA were added to each reaction. The cycling conditions were set as $10 \mathrm{~min}$ of preliminary denaturation at $95{ }^{\circ} \mathrm{C}$, then 40 cycles of $15 \mathrm{~s}$ for denaturation at $94{ }^{\circ} \mathrm{C}, 60 \mathrm{~s}$ for primer annealing at $50{ }^{\circ} \mathrm{C}$, and $2 \mathrm{~min}$ for extension at $72{ }^{\circ} \mathrm{C}$, and finally $1 \mathrm{~min}$ at $72{ }^{\circ} \mathrm{C}$ as a terminal extension phase. Data were analyzed using the software accompanying the ABI7500 real-time PCR device V.2.0.1. Allele discrimination plots were constructed for MECP2 and TIRAP, and are shown in Supplementary Figure S1a,b, respectively.

\subsection{Sequencing of the MECP2 rs2734647and TIRAP rs8177374 Genes}

Genotyping of the MECP2 rs2734647and TIRAP rs8177374 polymorphisms was further confirmed by sequencing. The Gene JET PCR Purification Kit (THERMO SCIENTIFIC, Lot 00424822, Lithuania) was used in the first step of DNA purification prior to the sequencing step. The primer sequences of the MECP2 rs2734647 gene were F: AGCTTAAGCAAAGGAAATCTGG; R: GCTTTTCCCTGGGGATTG, while the primer sequences of the TIRAP rs8177374 gene were F: GTGTCTGGCCCTAATCTCATGAGGAAT; R: GCACTACACTCAGGAACACAGCAGAGTC. Afterwards, the samples were injected in cycler sequence after dilution with the primers to reach $10 \mathrm{pmol} / \mathrm{uL}$, and the PCR product was 
diluted to reach $20 \mathrm{ng}$ under the following thermal cycling conditions: $1 \mathrm{~min}$ at $96{ }^{\circ} \mathrm{C}$, then 25 cycles of $10 \mathrm{~s}$ at $94{ }^{\circ} \mathrm{C}, 5 \mathrm{~s}$ for primer annealing at $61{ }^{\circ} \mathrm{C}$, and $4 \mathrm{~min}$ for extension at $60{ }^{\circ} \mathrm{C}$, followed by a pause at $4{ }^{\circ} \mathrm{C}$, and then the CENTRI-SEP Kit (Princeton Separations, Adelphia, Lot: 12D7662) was used in the second purification step by applying heat shock to the samples with the Hi-Di reagent. The cycling conditions were $95^{\circ} \mathrm{C}$ for $3 \mathrm{~min}$ followed by holding at $4{ }^{\circ} \mathrm{C}$. Subsequently, samples were immediately stored at $-20{ }^{\circ} \mathrm{C}$ for 2 min and frozen. The samples were analyzed using an AB Applied Biosystems HITACHI 3500 genetic analyzer. MECP2 sequence analysis is demonstrated in Supplementary Figure S1c, whereas TIRAP sequence analysis is shown in Supplementary Figure S1d.

\subsection{Statistical Analysis}

IBM SPSS software package version 20.0 (IBM Corp., Armonk, NY, USA:) was used for data analysis. Data were evaluated by means of the Kolmogorov-Smirnov test to verify the normality of the distribution of variables. Quantitative data were analyzed as the mean and standard deviation $(X \pm S D)$. Qualitative data were set as number and percentage (No and $\%$ ) and analyzed using the chi-squared test. The significance of the obtained results was decided at $p<0.05$.

\section{Results}

Data from all 210 participants in the current study (110 SLE patients and 100 healthy controls) were analyzed statistically, and the results are shown in Tables 1 and 2. The sample size was calculated to be 210 (110 cases and 100 controls), taking power at $80 \%$ and the significance level at 0.05 , minor allele frequency in the control group of $5.1 \%$, and an average odds ratio of 4.21. Age was matched between groups, where the mean age of the patients was (37.95 \pm 9$)$. Patients had a significant average visual analogue scale (VAS) of $30(p<0.001)$. Arthralgia was predominant in $63(57.3 \%)$, while arthritis was detected in only $17(15.5 \%)$, and 30 (27.35\%) had no joint affection. Systolic blood pressure (SBP) was significantly different between groups $(p<0.001)$, while diastolic blood pressure (DBP) did not significantly vary $(p=0.221)$ (Table 1$)$. Vitamin A was significantly lower in patients than in healthy controls $(p<0.001)$. Immune markers including regulatory T cells (Treg \%) and complements three (C3) and four (C4) were markedly lower in patients than in controls $(p<0.001)$, whereas T helper 17 (Th17\%) was considerably elevated in patients compared to controls $(p<0.001)$. Markers of inflammation-including erythrocyte sedimentation rate (ESR) and C-reactive protein (CRP) — were demonstrably increased in patients $(p<0.001)$, indicating the presence of active disease. Elements of $\mathrm{CBC}$-including $\mathrm{Hb}$ and $\mathrm{WBC}$ were significantly higher in patients $(p<0.001)$, while platelets did not vary significantly between groups $(p=0.076)$. Renal functions were elevated in patients, indicating kidney involvement, where serum urea $(p=0.001)$, serum creatinine $(p<0.001)$, and albumincreatinine ratio $(\mathrm{ACR})(p<0.001)$ were all markedly elevated in patients compared to controls. The mean SLEDAI in patients was $(6.4 \pm 2.8)$, proving disease activity, while the mean anti-ds-DNA titer was relatively high $(88.23 \pm 60.71)$. Treatment of SLE varied between patients, where all patients (100\%) received azathioprine, 47 patients $(42.7 \%)$ were given chloroquine phosphate and corticosteroids, while mycophenolate mofetil was given to $27(24.5 \%)$, and only $10(9.1 \%)$ patients were treated with cyclophosphamide (Table 1$)$.

The genotype and allele distribution of MECP2 rs2734647 and TIRAP rs8177374 were investigated, and the Hardy-Weinberg equilibrium was analyzed. Neither SNP deviated from the Hardy-Weinberg equilibrium, where MECP2 rs2734647 was 0.052 in patients and 0.067 in controls, while TIRAP rs 8177374 was 0.626 in patients and 0.266 in controls. 
Table 1. Comparison between the two studied groups according to different parameters.

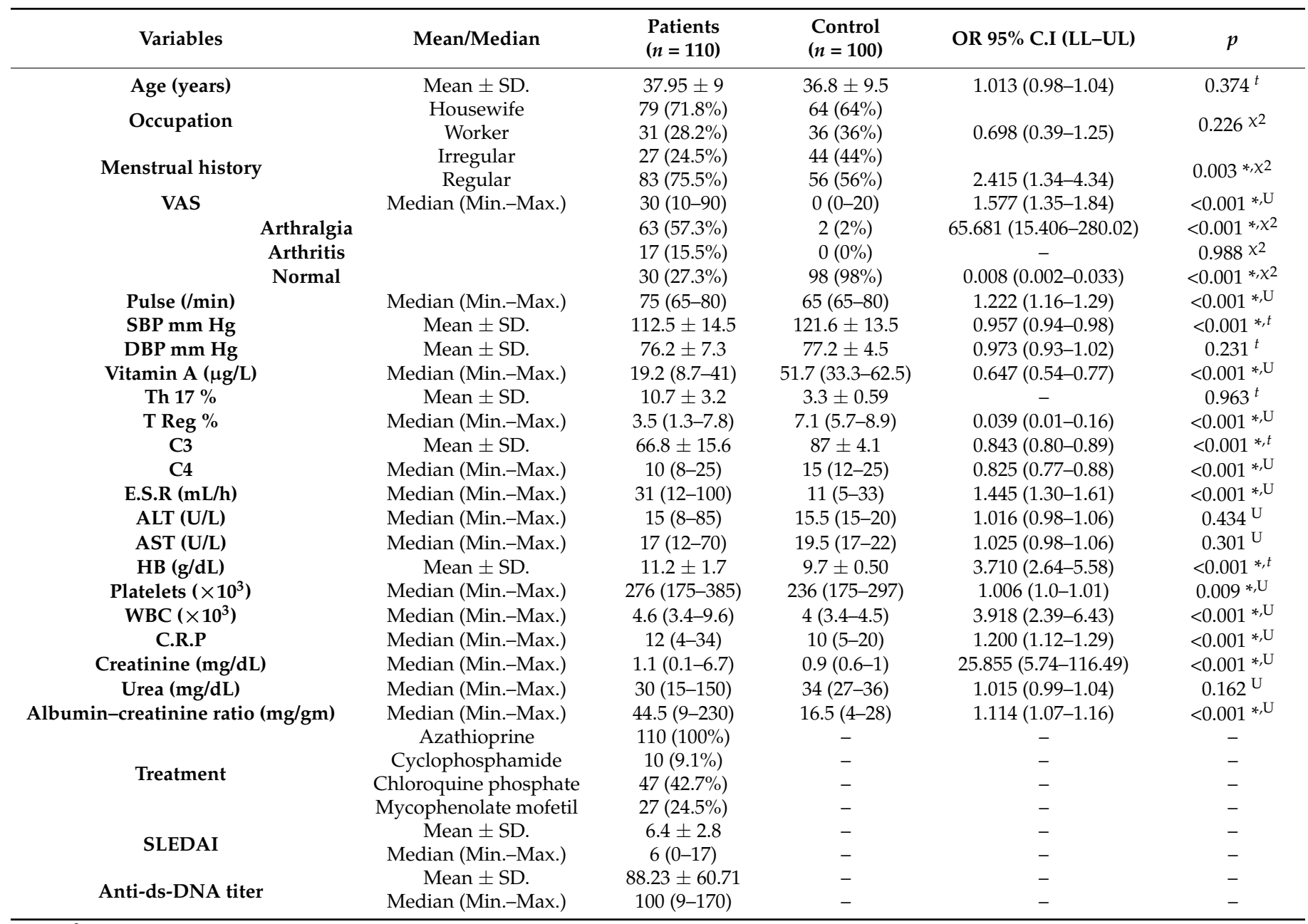

$\chi^{2}$ : Chi-squared test; ${ }^{t}$ : Student's $t$-test; ${ }^{U}$ : Mann-Whitney test; $p$ : $p$-value for comparing between the studied groups; ${ }^{*}$ statistically significant at $p \leq 0.05$. Visual analogue scale (VAS); erythrocyte sedimentation rate (ESR); albumin-creatinine ratio (ACR); alanine aminotransferase (ALT); and aspartate aminotransferase (AST); C-reactive protein (highly sensitive) (CRP); anti-double-stranded-DNA titer (anti-ds-DNA); and complement C3 and C4 systemic lupus erythematosus disease activity index (SLEDAI).

Table 2. Comparison between the two studied groups according to MECP2 rs2734647 and TIRAP rs8177374.

\begin{tabular}{|c|c|c|c|c|}
\hline Gene & $\begin{array}{l}\text { Patients } \\
(n=110)\end{array}$ & $\begin{array}{c}\text { Control } \\
(n=100)\end{array}$ & OR (95\% C.I) (LL-UL) & $p$ \\
\hline \multicolumn{5}{|c|}{$M E C P 2$ rs2734647 } \\
\hline $\mathrm{CC}$ & $65(59.1 \%)$ & $81(81 \%)$ & 1.000 & \\
\hline CT & $34(30.9 \%)$ & $16(16 \%)$ & $2.648 *(1.34-5.22)$ & $0.005 *$ \\
\hline TT & $11(10 \%)$ & $3(3 \%)$ & $4.569(1.22-17.06)$ & $0.024 *$ \\
\hline$(\mathrm{CT}+\mathrm{TT})$ & $45(40.9 \%)$ & $19(19 \%)$ & $2.951(1.58-5.53)$ & $0.001 *$ \\
\hline HWE & 0.052 & $\begin{array}{c}0.067 \\
\text { Allele }\end{array}$ & & \\
\hline $\mathrm{C}$ & $164(74.5 \%)$ & $178(89 \%)$ & 1.000 & \\
\hline $\mathbf{T}$ & $56(25.5 \%)$ & $\begin{array}{c}22(11 \%) \\
\text { TIRAP rs8177374 }\end{array}$ & $2.763(1.615-4.726)$ & $<0.001$ * \\
\hline $\mathrm{CC}$ & $64(58.2 \%)$ & $82(82 \%)$ & 1.000 & \\
\hline CT & $41(37.3 \%)$ & $16(16 \%)$ & $3.283(1.690-6.377)$ & $<0.001$ * \\
\hline TT & $5(4.5 \%)$ & $2(2 \%)$ & $3.203(0.602-17.051)$ & 0.172 \\
\hline$(\mathrm{CT}+\mathrm{TT})$ & $45(40.9 \%)$ & $18(18 \%)$ & $3.274(1.73-6.18)$ & $<0.001 *$ \\
\hline HWE & 0.626 & $\begin{array}{c}0.266 \\
\text { Allele }\end{array}$ & & \\
\hline $\mathrm{C}$ & $169(76.8 \%)$ & $180(90 \%)$ & 1.000 & \\
\hline $\mathbf{T}$ & $51(23.2 \%)$ & $20(10 \%)$ & $2.716 *(1.554-4.746)$ & $<0.001 *$ \\
\hline
\end{tabular}

OR: odds ratio; LL: lower limit; UL: upper limit; C.I: confidence interval; *: statistically significant at $p \leq 0.05$

HWE: Hardy-Weinberg equilibrium. 
The presence of the T allele of MECP2 rs2734647 increases susceptibility to the disease where the individual CT and TT genotypes increase the risk of SLE with an odds ratio (OR) of $2.648(p=0.005)$ and $4.569(p=0.024)$, respectively, while having both (CT and TT) increased the risk with an OR of $2.951(p=0.001)$. T allele frequency was $25.5 \%$ in patients, which was markedly higher than controls $(11 \%)$, with an OR of $2.763(p<0.001)$. Moreover, we noticed that the CT and TT genotypes of TIRAP rs8177374 increased the risk of SLE with an OR of $3.283(p<0.001)$ and $3.203(p=0.172)$, respectively, while having both (CT and TT) increased the risk with an OR of $3.274(p<0.001)$. T allele frequency was $23.2 \%$ in patients; however, in controls, it was only 10\%, with an OR of $2.716(p<0.001)$ (Table 2).

We further classified 110 SLE patients based on renal affection into SLE with nephritis $(n=63)$ and SLE without nephritis $(n=47)$, and compared both subgroups with controls, as shown in Tables 3 and 4 . Age was matched between groups $(p=0.094)$. Occupation $(p=0.001)$ and menstrual history $(p=0.002)$ were significantly different between groups, where workers represented $41.3 \%$ of the nephritis group but only $10.6 \%$ of the non-nephritis group. Moreover, $82.5 \%$ of nephritis patients had regular menstruation, compared to $66 \%$ in the non-nephritis group and 56\% of controls. Furthermore, VAS was markedly elevated in the nephritis group, with an average of 30, while in the non-nephritis group it was 20 and in controls it was $0(p<0.001)$, showing significant differences between groups. Concerning affected joints, arthralgia was evident in 54\% of the nephritis group, while being a more common manifestation in the non-nephritis group $(61.7 \%)(p<0.001)$, and arthritis was more prevalent in the nephritis group (19\%) than in the non-nephritis group $(10.6 \%)$ $(p<0.001)$. Systolic $(p<0.001)$ and diastolic BP $(p=0.019)$ were significantly different between groups; in addition, both were higher in the nephritis than the non-nephritis group $(p=0.029)$.

Vitamin A was noticeably lower in the nephritis group than in the non-nephritis and control groups $(p<0.001)$. The SLEDAI was distinctly higher in nephritis patients than in non-nephritis patients $(p<0.001)$, demonstrating more active disease in the former, although the anti-ds-DNA titer was to some extent equal in both groups $(p=0.508)$. Standard management included azathioprine, which was given to all patients; cyclophosphamide and mycophenolate mofetil were given to nephritis patients only $(p=0.005, p<0.001$, respectively), while chloroquine phosphate was taken by $41.3 \%$ of nephritis patients and $44.7 \%$ of non-nephritis patients $(p=0.721)$ (Table 3$)$.

Table 3. Comparisons between the three studied groups according to different parameters.

\begin{tabular}{|c|c|c|c|c|c|}
\hline \multirow[b]{2}{*}{ Variables } & \multicolumn{2}{|c|}{ SLE Patients $(n=110)$} & \multirow[b]{2}{*}{$\begin{array}{l}\text { Control } \\
(n=100)\end{array}$} & \multirow[b]{2}{*}{ Test of Sig. } & \multirow[b]{2}{*}{$p$} \\
\hline & $\begin{array}{l}\text { With Nephritis } \\
\quad(n=63)\end{array}$ & $\begin{array}{l}\text { Without Nephritis } \\
\quad(n=47)\end{array}$ & & & \\
\hline Occupation & $36.4 \pm 8.9$ & $40 \pm 8.8$ & $36.8 \pm 9.5$ & $\mathrm{~F}=2.395$ & 0.094 \\
\hline $\begin{array}{c}\text { Housewife } \\
\text { Worker }\end{array}$ & $\begin{array}{l}37(58.7 \%) \\
26(41.3 \%)\end{array}$ & $\begin{array}{l}42(89.4 \%) \\
5(10.6 \%)\end{array}$ & $\begin{array}{l}64(64 \%) \\
36(36 \%)\end{array}$ & \multirow[t]{2}{*}{$\chi^{2}=13.099 *$} & $0.001 *$ \\
\hline \multicolumn{5}{|l|}{ Menstrual History } & \\
\hline $\begin{array}{l}\text { Irregular } \\
\text { Regular }\end{array}$ & $\begin{array}{l}11(17.5 \%) \\
52(82.5 \%)\end{array}$ & $\begin{array}{l}16(34 \%) \\
31(66 \%)\end{array}$ & $\begin{array}{l}44(44 \%) \\
56(56 \%)\end{array}$ & $\chi^{2}=12.166 *$ & $0.002 *$ \\
\hline VAS Median (Min.-Max.) & $30(10-90)$ & $20(10-70)$ & $0(0-20)$ & & 0.002 \\
\hline Sig. bet. grps. & \multicolumn{3}{|c|}{$p_{1}=0.048 *, p_{2}<0.001 *, p_{3}=0.001 *$} & & \\
\hline Arthralgia & $34(54 \%)$ & $29(61.7 \%)$ & $2(2 \%)$ & $\chi^{2}=75.631 *$ & $<0.001 *$ \\
\hline Arthritis & $12(19 \%)$ & $5(10.6 \%)$ & $0(0 \%)$ & $\chi^{2}=19.374 *$ & $<0.001 *$ \\
\hline Normal & $17(27 \%)$ & $13(27.7 \%)$ & $98(98 \%)$ & $\chi^{2}=110.099 *$ & $<0.001 *$ \\
\hline
\end{tabular}


Table 3. Cont.

\begin{tabular}{|c|c|c|c|c|c|}
\hline \multirow[b]{2}{*}{ Variables } & \multicolumn{2}{|c|}{ SLE Patients $(n=110)$} & \multirow[b]{2}{*}{$\begin{array}{l}\text { Control } \\
(n=100)\end{array}$} & \multirow[b]{2}{*}{ Test of Sig. } & \multirow[b]{2}{*}{$p$} \\
\hline & $\begin{array}{l}\text { With Nephritis } \\
\qquad(n=63)\end{array}$ & $\begin{array}{l}\text { Without Nephritis } \\
\qquad(n=47)\end{array}$ & & & \\
\hline Pulse Median (Min.-Max.) & $75(65-80)$ & $80(65-80)$ & $65(65-80)$ & $\mathrm{K}=52.130 *$ & $<0.001 *$ \\
\hline $\begin{array}{l}\text { Sig. bet. grps. } \\
\text { Systolic BP mmHg }\end{array}$ & \multicolumn{3}{|c|}{$p_{1}=0.110, p_{2}<0.001 *, p_{3}=0.001 *$} & & \\
\hline Mean \pm SD. & $115.4 \pm 16.2$ & $108.5 \pm 10.8$ & $121.6 \pm 13.5$ & $\mathrm{~F}=14.674 *$ & $<0.001 *$ \\
\hline $\begin{array}{l}\text { Sig. bet. grps. } \\
\text { Diastolic BP mmHg }\end{array}$ & \multicolumn{3}{|c|}{$p_{1}=0.029^{*}, p_{2}=0.016^{*}, p_{3}<0.001 *$} & & \\
\hline Mean \pm SD. & $77.5 \pm 8.4$ & $74.5 \pm 5$ & $77.2 \pm 4.5$ & $\mathrm{~F}=4.037^{*}$ & $0.019 *$ \\
\hline Sig. bet. grps. & \multicolumn{3}{|c|}{$p_{1}=0.029 *, p_{2}=0.961, p_{3}=0.030 *$} & & \\
\hline $\begin{array}{c}\text { Vitamin A ( } \mu \mathrm{g} / \mathrm{L}) \\
\text { Median (Min.-Max.) }\end{array}$ & $17.8(8.7-41)$ & $33.7(8.7-41)$ & $51.7(33.3-62.5)$ & $\mathrm{K}=155.791$ * & $<0.001 *$ \\
\hline $\begin{array}{l}\text { Sig. bet. grps. } \\
\text { Treatment }\end{array}$ & \multicolumn{3}{|c|}{$p_{1}=0.004^{*}, p_{2}<0.001^{*}, p_{3}<0.001^{*}$} & & \\
\hline Azathioprine & $63(100 \%)$ & $47(100 \%)$ & - & - & - \\
\hline Cyclophosphamide & $10(15.9 \%)$ & $0(0 \%)$ & - & $\chi^{2}=8.206$ & $\mathrm{FE}_{p}=0.005$ * \\
\hline Chloroquine phosphate & $26(41.3 \%)$ & $21(44.7 \%)$ & - & $\chi^{2}=0.128$ & 0.721 \\
\hline Mycophenolate mofetil & $27(42.9 \%)$ & $0(0 \%)$ & - & $\chi^{2}=26.695$ & $<0.001 *$ \\
\hline SLEDAIMedian (Min.-Max.) & $6(5-17)$ & $5(0-11)$ & - & & \\
\hline $\begin{array}{c}\text { Anti-ds-DNAtiter Median (Min.-Max.) } \\
\text { Th17\% }\end{array}$ & $100(9-170)$ & $100(9-170)$ & - & & \\
\hline Mean \pm SD. & $11.8 \pm 2.7$ & $9.2 \pm 3.2$ & $3.3 \pm 0.59$ & $\mathrm{~F}=322.570 *$ & $<0.001 *$ \\
\hline $\begin{array}{l}\text { Sig. bet. grps. } \\
\text { Treg } \%\end{array}$ & \multicolumn{3}{|c|}{$p_{1}<0.001^{*}, p_{2}<0.001^{*}, p_{3}<0.001^{*}$} & & \\
\hline Median (Min.-Max.) & $2.8(1.3-6.3)$ & $4.8(2.1-7.8 /)$ & $7.1(5.7-8.9)$ & $\mathrm{K}=152.721 *$ & $<0.001 *$ \\
\hline $\begin{array}{l}\text { Sig. bet. grps. } \\
\text { C3 }\end{array}$ & \multicolumn{3}{|c|}{$p_{1}<0.001^{*}, p_{2}<0.001^{*}, p_{3}=0.445$} & & \\
\hline $\begin{array}{l}\text { Mean } \pm \text { SD. } \\
\text { Sig. bet. grps. }\end{array}$ & \multicolumn{3}{|c|}{$\begin{array}{c}58.3 \pm 10.3 \\
p_{1}<0.001 *\end{array} p_{2}<0.001 *, p_{3}<0.001 * * 4.1$} & $\mathrm{~F}=187.862 *$ & $<0.001 *$ \\
\hline $\begin{array}{c}\text { C4 } \\
\text { Median (Min.-Max.) }\end{array}$ & $9(8-20)$ & $16(9-25)$ & $15(12-25)$ & $\mathrm{K}=104.050 *$ & $<0.001 *$ \\
\hline $\begin{array}{l}\text { Sig. bet. grps. } \\
\text { E.S.R }(\mathrm{mL} / \mathrm{h})\end{array}$ & \multicolumn{3}{|c|}{$p_{1}<0.001^{*}, p_{2}<0.001^{*}, p_{3}=0.445$} & & \\
\hline Median (Min.-Max.) & $32(12-100)$ & $30(22-75)$ & $11(5-33)$ & $\mathrm{K}=138.457$ * & $<0.001 *$ \\
\hline $\begin{array}{l}\text { Sig. bet. grps. } \\
\text { ALT (U/L) }\end{array}$ & \multicolumn{3}{|c|}{$p_{1}=0.960, p_{2}<0.001^{*}, p_{3}<0.001^{*}$} & & \\
\hline Median (Min.-Max.) & $14(8-85)$ & $15(10-23)$ & 15.5(15-20) & $\mathrm{K}=16.218^{*}$ & $<0.001 *$ \\
\hline $\begin{array}{l}\text { Sig. bet. grps. } \\
\text { AST(U/L) }\end{array}$ & \multicolumn{3}{|c|}{$p_{1}=0.004^{*}, p_{2}<0.001 *, p_{3}=0.666$} & & \\
\hline Median (Min.-Max.) & $17(12-70)$ & $17(12-32)$ & $19.5(17-22)$ & $\mathrm{K}=12.684$ * & $0.004 *$ \\
\hline $\begin{array}{l}\text { Sig. bet. grps. } \\
\text { HB (gm/dL) }\end{array}$ & \multicolumn{3}{|c|}{$p_{1}=0.003^{*}, p_{2}=0.001^{*}, p_{3}=0.756$} & & \\
\hline $\begin{array}{l}\text { Mean } \pm \text { SD. } \\
\text { Sig. bet. grps. } \\
\text { Platelets }\left(\times 10^{3}\right)\end{array}$ & \multicolumn{3}{|c|}{$\begin{array}{c}10.8 \pm 1.3 \\
p_{1}<0.001 *, p_{2}<0.001 *, p_{3}<0.001 *\end{array}$} & $\mathrm{~F}=47.360 *$ & $<0.001 *$ \\
\hline Median (Min.-Max.) & $262(175-385)$ & $276(175-332)$ & $236(175-297)$ & $K=3.163$ & 0.206 \\
\hline $\begin{array}{l}\text { WBC }\left(\times 10^{3}\right) \\
\text { Median (Min.-Max.) } \\
\text { Sig. bet. grps. }\end{array}$ & $\begin{array}{l}4.5(3.4-9.6) \\
p_{1}=0\end{array}$ & $\begin{array}{c}4.6(3.4-7) \\
2 *, p_{2}<0.001 *, p_{3}<\end{array}$ & $4(3.4-4.5)$ & $\mathrm{K}=75.729 *$ & $<0.001 *$ \\
\hline $\begin{array}{c}\text { C.R.P } \\
\text { Median (Min.-Max.) }\end{array}$ & $12(5-34)$ & $12(4-25)$ & $10(5-20)$ & $K=25.056^{*}$ & $<0.001 *$ \\
\hline $\begin{array}{l}\text { Sig. bet. grps. } \\
\text { Creatinine (mg/dL) } \\
\text { Median (Min.-Max.) } \\
\text { Sig. bet. grps. }\end{array}$ & $\begin{array}{r}1.2(1-6.7) \\
p_{1}<0\end{array}$ & $\begin{array}{c}0.9(0.10-1.90) \\
01^{*}, p_{2}<0.001^{*}, p_{3}=\end{array}$ & $\begin{array}{l}0.90(0.60-1) \\
750\end{array}$ & $\mathrm{~K}=112.959 *$ & $<0.001 *$ \\
\hline $\begin{array}{l}\text { Urea (mg/dL) } \\
\text { Median (Min.-Max.) } \\
\text { Sig. bet. grps. }\end{array}$ & \multicolumn{3}{|c|}{$\begin{array}{c}34(15-150) \\
p_{1}<0.001^{*}, p_{2}<0.001^{*}, p_{3}=0.418\end{array}$} & $\mathrm{~K}=24.559 *$ & $<0.001 *$ \\
\hline $\begin{array}{c}\text { Albumin-creatinine ratio (ACR) (mg/gm) } \\
\text { Median (Min.-Max.) } \\
\text { Sig. bet. grps. }\end{array}$ & \multicolumn{3}{|c|}{$\begin{array}{c}90(34-230) \\
p_{1}<0.001^{*}, p_{2}<0.001^{*}, p_{3}=0.104\end{array}$} & $\mathrm{~K}=132.840$ * & $<0.001 *$ \\
\hline
\end{tabular}

$\chi^{2}$ : chi-squared test; FE: Fisher's exact test; F: F for ANOVA test, pairwise comparisons between groups were done using Tukey's post hoc test. K: K for Kruskal-Wallis test, pairwise comparisons between groups were done using Dunn's post hoc test for multiple comparisons. $p$ : $p$-value for comparing between the studied groups. $p_{1}: p$-value for comparing between patients with nephritis and those without nephritis. $p_{2}: p$-value for comparing between patients with nephritis and controls. $p_{3}$ : $p$-value for comparing between patients without nephritis and controls. *: Statistically significant at $p \leq 0.05$. 
Table 4. Comparison between the three studied groups according to MECP2 rs2734647 and TIRAP rs8177374.

\begin{tabular}{|c|c|c|c|c|c|c|c|c|c|}
\hline Gene & $\begin{array}{l}\text { SLE with } \\
\text { Nephritis } \\
(n=63)\end{array}$ & $\begin{array}{l}\text { SLE without } \\
\text { Nephritis } \\
(n=47)\end{array}$ & $\begin{array}{c}\text { Control } \\
(n=100)\end{array}$ & $p_{1}$ & $\begin{array}{c}\mathrm{OR}_{1} \\
\text { (CI. 95\%) }\end{array}$ & $p_{2}$ & $\begin{array}{c}\mathrm{OR}_{2} \\
\text { (CI. 95\%) }\end{array}$ & $p_{3}$ & $\begin{array}{c}\mathrm{OR}_{3} \\
\text { (CI. 95\%) }\end{array}$ \\
\hline \multicolumn{10}{|c|}{ MECP2 rs2734647 } \\
\hline $\mathrm{CC}$ & $\begin{array}{c}31 \\
(49.2 \%)\end{array}$ & $\begin{array}{c}34 \\
(72.3 \%)\end{array}$ & $\begin{array}{c}81 \\
(81 \%)\end{array}$ & & 1.000 & & 1.000 & & 1.000 \\
\hline CT & $\begin{array}{c}22 \\
(34.9 \%)\end{array}$ & $\begin{array}{c}12 \\
(25.5 \%)\end{array}$ & $\begin{array}{c}16 \\
(16 \%)\end{array}$ & 0.109 & $\begin{array}{c}2.01 \\
(0.86-4.73)\end{array}$ & $0.001 *$ & $\begin{array}{c}3.593 \\
(1.67-7.73)\end{array}$ & 0.180 & $\begin{array}{c}1.787 \\
(0.76-4.18)\end{array}$ \\
\hline TT & $\begin{array}{c}10 \\
(15.9 \%)\end{array}$ & $\begin{array}{c}1 \\
(2.1 \%)\end{array}$ & $\begin{array}{c}3 \\
(3 \%)\end{array}$ & $0.026 *$ & $\begin{array}{c}10.97 \\
(1.33-90.69)\end{array}$ & $0.002 *$ & $\begin{array}{c}8.710 \\
(2.25-33.77)\end{array}$ & 0.844 & $\begin{array}{c}0.794 \\
(0.08-7.91)\end{array}$ \\
\hline$(\mathrm{CT}+\mathrm{TT})$ & $\begin{array}{c}32 \\
(50.8 \%)\end{array}$ & $\begin{array}{c}13 \\
(27.7 \%)\end{array}$ & $\begin{array}{c}19 \\
(19 \%)\end{array}$ & $0.016^{*}$ & $\begin{array}{c}2.700 \\
(1.20-6.06)\end{array}$ & $<0.001 *$ & $\begin{array}{c}4.401 \\
(2.18-8.88)\end{array}$ & 0.238 & $\begin{array}{c}1.630 \\
(0.72-3.67)\end{array}$ \\
\hline \multicolumn{10}{|c|}{ Allele } \\
\hline $\mathrm{C}$ & $\begin{array}{c}84 \\
(66.7 \%)\end{array}$ & $\begin{array}{c}80 \\
(85.1 \%)\end{array}$ & $\begin{array}{c}178 \\
(89 \%)\end{array}$ & & 1.000 & & 1.000 & & 1.000 \\
\hline $\mathbf{T}$ & $\begin{array}{c}42 \\
(33.3 \%)\end{array}$ & $\begin{array}{c}14 \\
(14.9 \%)\end{array}$ & $\begin{array}{c}22 \\
(11 \%)\end{array}$ & & $\begin{array}{c}2.857 \\
(1.45-5.63)\end{array}$ & $<0.001 *$ & $\begin{array}{c}4.045 \\
(2.27-7.21)\end{array}$ & 0.344 & $\begin{array}{c}1.416 \\
(0.69-2.90)\end{array}$ \\
\hline \multicolumn{10}{|c|}{ TIRAP rs8177374 } \\
\hline $\mathrm{CC}$ & $\begin{array}{c}29 \\
(46 \%)\end{array}$ & $\begin{array}{c}35 \\
(74.5 \%)\end{array}$ & $\begin{array}{c}82 \\
(82 \%)\end{array}$ & & 1.000 & & 1.000 & & 1.000 \\
\hline CT & $\begin{array}{c}30 \\
(47.6 \%)\end{array}$ & $\begin{array}{c}11 \\
(23.4 \%)\end{array}$ & $\begin{array}{c}16 \\
(16 \%)\end{array}$ & $0.006^{*}$ & $\begin{array}{c}3.292 \\
(1.41-7.69)\end{array}$ & $<0.001 *$ & $\begin{array}{c}5.302 \\
(2.53-11.11)\end{array}$ & 0.279 & $\begin{array}{c}1.611 \\
(0.68-3.82)\end{array}$ \\
\hline TT & $\begin{array}{c}4 \\
(6.3 \%)\end{array}$ & $\begin{array}{c}1 \\
(2.1 \%)\end{array}$ & $\begin{array}{c}2 \\
(2 \%)\end{array}$ & 0.169 & $\begin{array}{c}4.828 \\
(0.51-45.62)\end{array}$ & 0.052 & $\begin{array}{c}5.655 \\
(0.98-32.52)\end{array}$ & 0.899 & $\begin{array}{c}1.171 \\
(0.10-13.34)\end{array}$ \\
\hline$(\mathrm{CT}+\mathrm{TT})$ & $\begin{array}{c}34 \\
(54 \%)\end{array}$ & $\begin{array}{c}12 \\
(25.5 \%)\end{array}$ & $\begin{array}{c}18 \\
(18 \%)\end{array}$ & $0.003 *$ & $\begin{array}{c}3.420 \\
(1.50-7.78)\end{array}$ & $<0.001 *$ & $\begin{array}{c}5.341 \\
(2.6-10.88)\end{array}$ & 0.293 & $\begin{array}{c}1.562 \\
(0.68-3.58)\end{array}$ \\
\hline \multicolumn{10}{|c|}{ Allele } \\
\hline $\mathrm{C}$ & $\begin{array}{c}88 \\
(69.8 \%)\end{array}$ & $\begin{array}{c}81 \\
(86.2 \%)\end{array}$ & $\begin{array}{c}180 \\
(90 \%)\end{array}$ & & 1.000 & & 1.000 & & 1.000 \\
\hline $\mathbf{T}$ & $\begin{array}{c}38 \\
(30.2 \%)\end{array}$ & $\begin{array}{c}13 \\
(13.8 \%)\end{array}$ & $\begin{array}{c}20 \\
(10 \%)\end{array}$ & $0.005 *$ & $\begin{array}{c}2.691 \\
(1.34-5.41)\end{array}$ & $<0.001 *$ & $\begin{array}{c}3.886 \\
(2.14-7.07)\end{array}$ & 0.334 & $\begin{array}{c}1.444 \\
(0.68-3.04)\end{array}$ \\
\hline
\end{tabular}

$\mathrm{OR}_{1}$ : Odds ratio for patients with and without nephritis. $\mathrm{OR}_{2}$ : Odds ratio for patients with nephritis and controls. OR ${ }_{3}$ : Odds ratio for patients without nephritis and controls. CI: confidence interval; LL: lower limit; UL: upper limit. *: Statistically significant at $p \leq 0.05$. $p_{1}$ : $p$ value for comparing between with nephritis and without nephritis. $p_{2}: p$ value for comparing between with nephritis and control. $p_{3}$ : $p$ value for comparing between without nephritis and control.

The immune markers Treg percentage, $\mathrm{C} 3$, and $\mathrm{C} 4$ were definitely lower in nephritis patients compared to non-nephritis patients $(p<0.001)$ and controls $(p<0.001)$. Conversely, Th17 percentage was clearly increased in nephritis patients compared with non-nephritis patients $(p<0.001)$ and controls $(p<0.001)$. Moreover, inflammatory markers including ESR and CRP were elevated in both nephritis $(p<0.001)$ and non-nephritis patients $(p<0.001$ and $p=0.002$, respectively) versus controls. Parameters of renal functions-including serum creatinine, serum urea, and ACR - were all prominently elevated in nephritis patients compared with non-nephritis patients $(p<0.001)$ and controls $(p<0.001)$, and the differences between non-nephritis patients and controls were not significant (Table 3).

MECP2 rs2734647 and TIRAP rs8177374 frequencies and dissemination were analyzed among subgroups, as shown in Table 4 . The $\mathrm{T}$ allele frequency of $M E C P 2 \mathrm{rs} 2734647$ was significantly higher in nephritis patients $(33.3 \%)$ than in non-nephritis patients $(14.9 \%$, OR1 $=2.857, p=0.002)$ or controls $(11 \%$, OR2 $=4.045, p<0.001)$, although the T allele difference between non-nephritis patients and controls was not significant (OR3 $=1.416$, $p=0.344$ ).

Furthermore, CT + TT of MECP2 was prominently elevated in nephritis patients $(50.8 \%)$ compared to non-nephritis patients $(27.7 \%$, OR1 $=2.70, p=0.016)$ and controls $(19 \%$, OR2 $=4.401, p<0.001)$, and no significant difference was noted between non-nephritis patients and controls $(\mathrm{OR} 3=1.63, p=0.238)$. Concerning TIRAP rs8177374, the T allele was prominently higher in nephritis patients $(30.2 \%)$ than in non-nephritis patients $(13.8 \%$, OR1 $=2.691, p=0.005)$ and controls $(10 \%$, OR2 $=3.886, p<0.001)$, although the T allele difference between non-nephritis patients and controls was not significant (OR3 $=1.444$, $p=0.334)$. 
Moreover, CT + TT of TIRAP was noticeably higher in nephritis patients (54.0\%) than in non-nephritis patients $(25.5 \%$, OR1 $=3.420, p=0.003)$ and controls $(18 \%, \mathrm{OR} 2=5.341$, $p<0.001$ ), and no significant distinction was observed between non-nephritis patients and controls (OR3 $=1.562, p=0.293)$ (Table 4).

The relationships of MECP2 rs2734647 genotypes CC $(n=31)$ and CT + TT $(n=32)$ with various parameters in SLE patients with nephritis are presented in Table 5. We observed that presence of the T allele in CT + TT was significantly associated with a higher ACR $(p<0.001)$ (Figure 1a), which might explain its association with increased risk of nephritis. Moreover, carriers of the $\mathrm{T}$ allele might show appropriate responses to cyclophosphamide, as nine patients $(28.1 \%)$ took this drug, while there was only one $(3.2 \%)$ carrier of the $\mathrm{C}$ allele in CC $(p=0.013)$, which might indicate that the $C$ allele can precipitate resistance to cyclophosphamide.

Table 5. Relationships between MECP2 rs2734647 genotypes and different parameters in SLE patients with nephritis $(n=63)$.

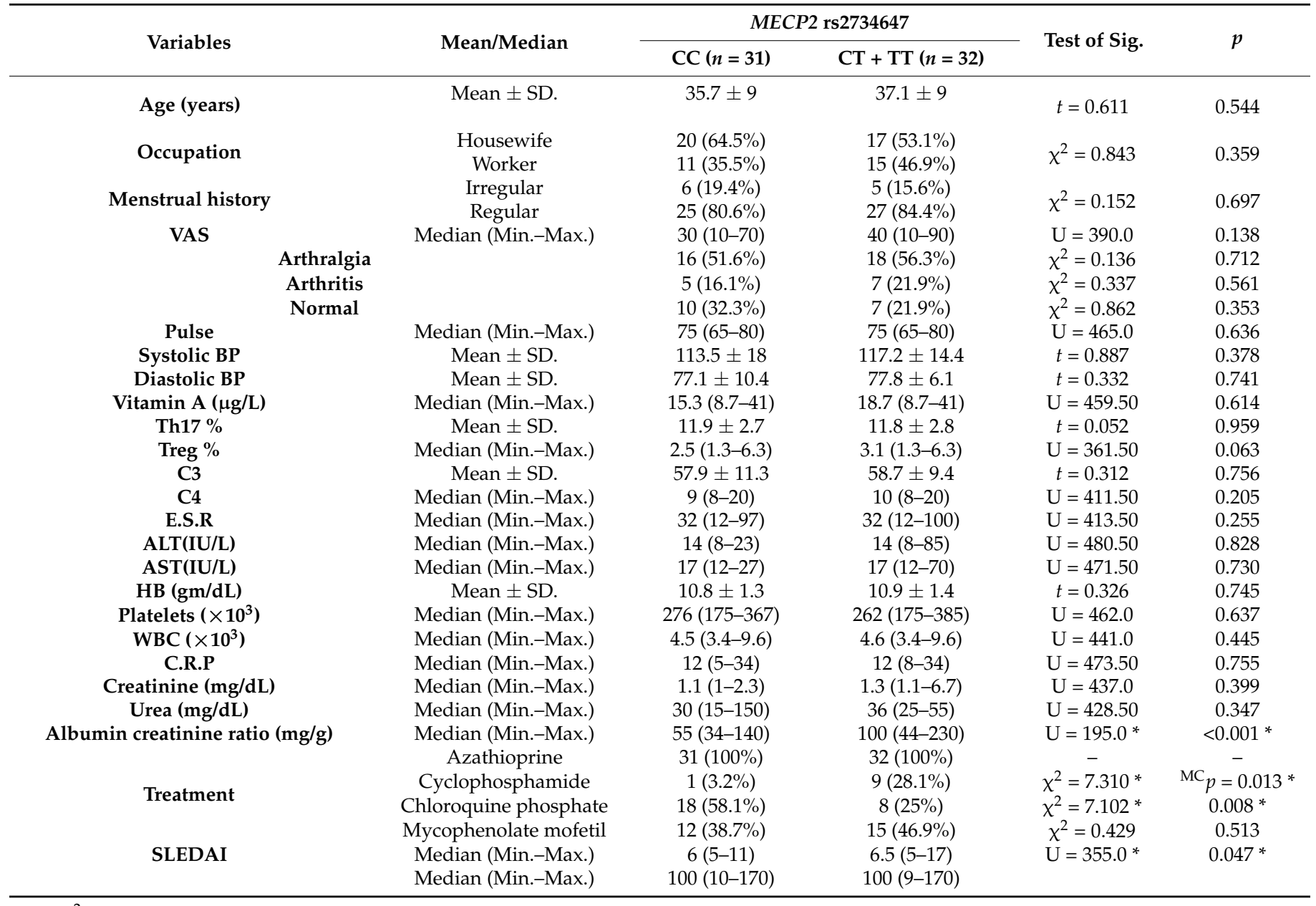

$\chi^{2}$ : chi-squared test; MC: Monte Carlo; $t$ : Student's $t$-test; U: Mann-Whitney test. $p$ : $p$-value for comparing between the studied groups. *: Statistically significant at $p \leq 0.05$. 
$\mathfrak{a}$

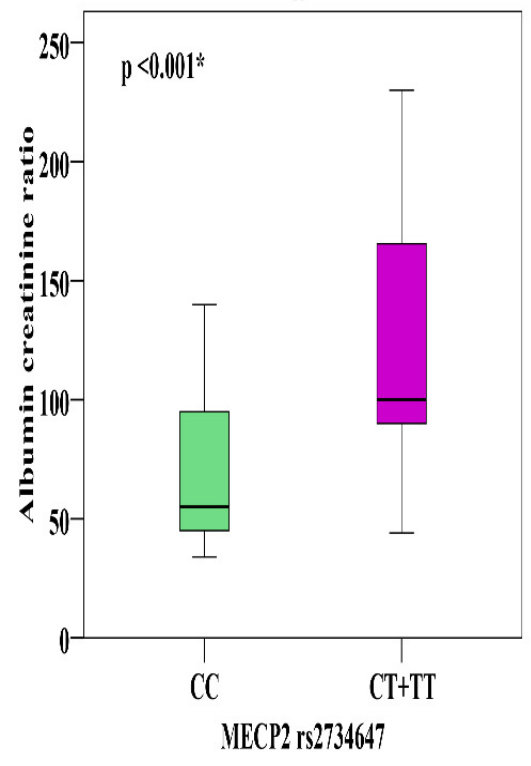

d

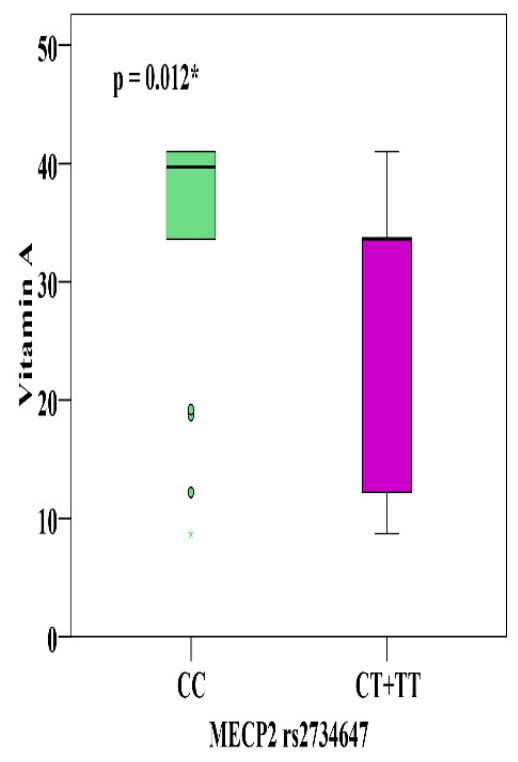

b

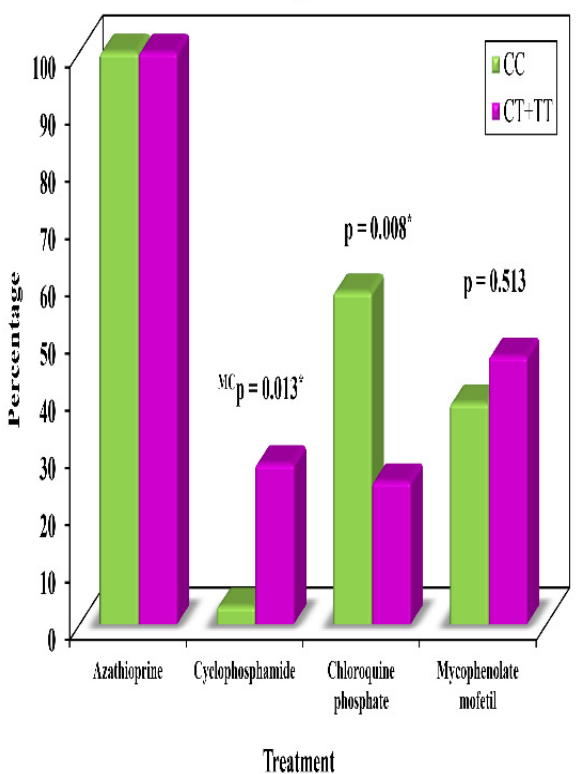

e

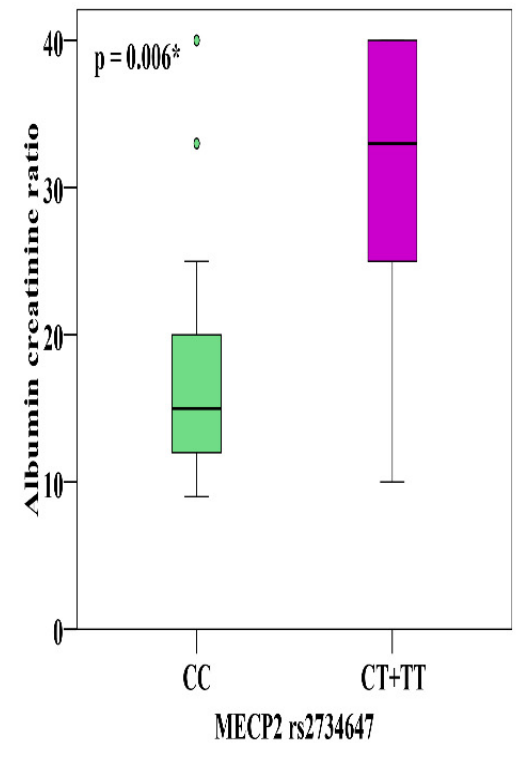

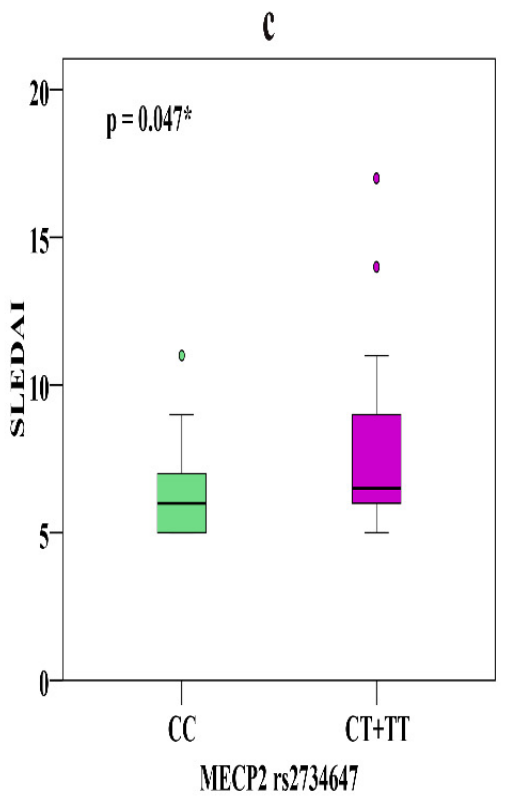

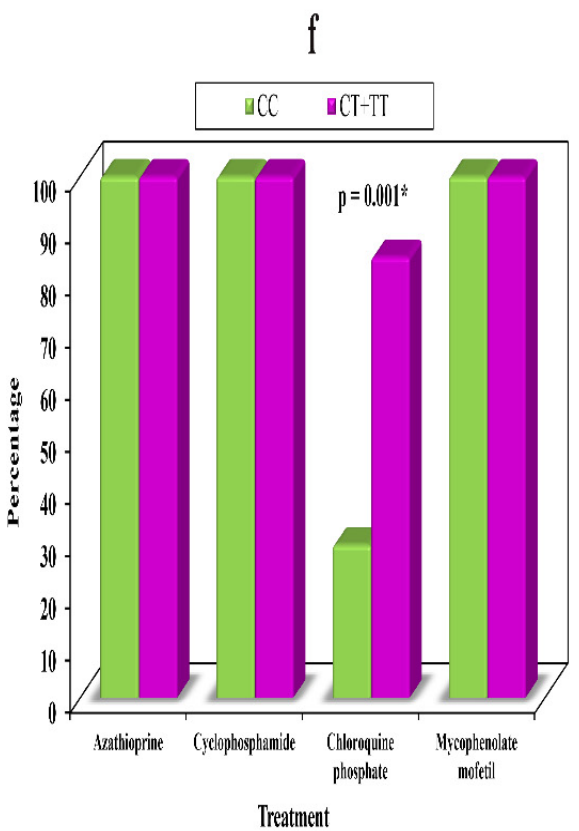

Figure 1. (a) Relationship between MECP2 rs2734647 and ACR in LN. (b) Relationship between MECP2 rs2734647 and treatment in LN. (c) Relationship between MECP2 rs2734647 and SLEDAI in LN. (d) Relationship between MECP2 rs2734647 and vitamin A in patients without nephritis. (e) Relationship between MECP2 rs2734647 and ACR in patients without nephritis. (f) Relationship between MECP2 rs2734647 and treatment in patients without nephritis. * — significant.

Conversely, carriers of the $\mathrm{C}$ allele showed a conventional response to chloroquine phosphate $18(58.1 \%)$ that was not evident in T allele carriers $8(25 \%)(p=0.008)$ (Figure $1 \mathrm{~b})$, which may prove that genetic background can determine the drug of choice in individual therapy. Carriers of the T allele showed higher SLEDAI scores $(p=0.047)$ than those carrying the $C$ allele (Figure 1c), which might explain a severe form of the disease (Table 5).

The relationships of MECP2 rs2734647 genotypes CC $(n=34)$ and CT + TT $(n=13)$ with various parameters in SLE patients without nephritis show that carriers of CT + TT have noticeably lower vitamin A levels $(p=0.012)$ (Figure 1d) and elevated ACR $(p=0.006)$ (Figure 1e) and showed better drug response to chloroquine phosphate $11(84.6 \%)$ than $C$ allele carriers $10(29.4 \%)(p=0.001)$ (Figure 1f). 
The relationships between TIRAP rs8177374 genotypes CC $(n=29)$ and CT + TT $(n=34)$ and various parameters in SLE patients with nephritis is presented in Table 6. Carriers of the T allele in CT + TT show noticeably higher ESR $(p=0.039)$ (Figure 2a) and ACR $(p=0.01)$ (Figure $2 b)$ than those carrying the $C$ allele which might illuminate its association with nephritis.

Carriers of the T allele showed evident response to cyclophosphamide in nine $(26.5 \%)$ patients, while only one $(3.4 \%)$ C allele carrier took cyclophosphamide $(p=0.016)$. However, chloroquine phosphate showed divergent results, with 18 (62.1\%) C allele carriers showing a therapeutic response, while only $8(23.5 \%)$ T allele carriers did so, suggesting that the latter might cause drug resistance $(p=0.002)$ (Figure 2c) (Table 6).

Concerning the relationships of TIRAP rs8177374 genotypes CC $(n=35)$ and CT + TT $(n=12)$, we observed that carriers of CT + TT showed markedly lower vitamin A levels $(p=0.041)$ (Figure 2d) and an elevated Th17 percentage $(p=0.04)$ (Figure 2e), along with a distinctly lower Treg percentage $(p=0.035)$ (Figure $2 \mathrm{f})$, compared with $C$ allele carriers. These significant relationships with immune markers might feature their association with disease risk.

Table 6. Relationships between TIRAP rs8177374 genotypes and different parameters in patients with nephritis $(n=63)$.

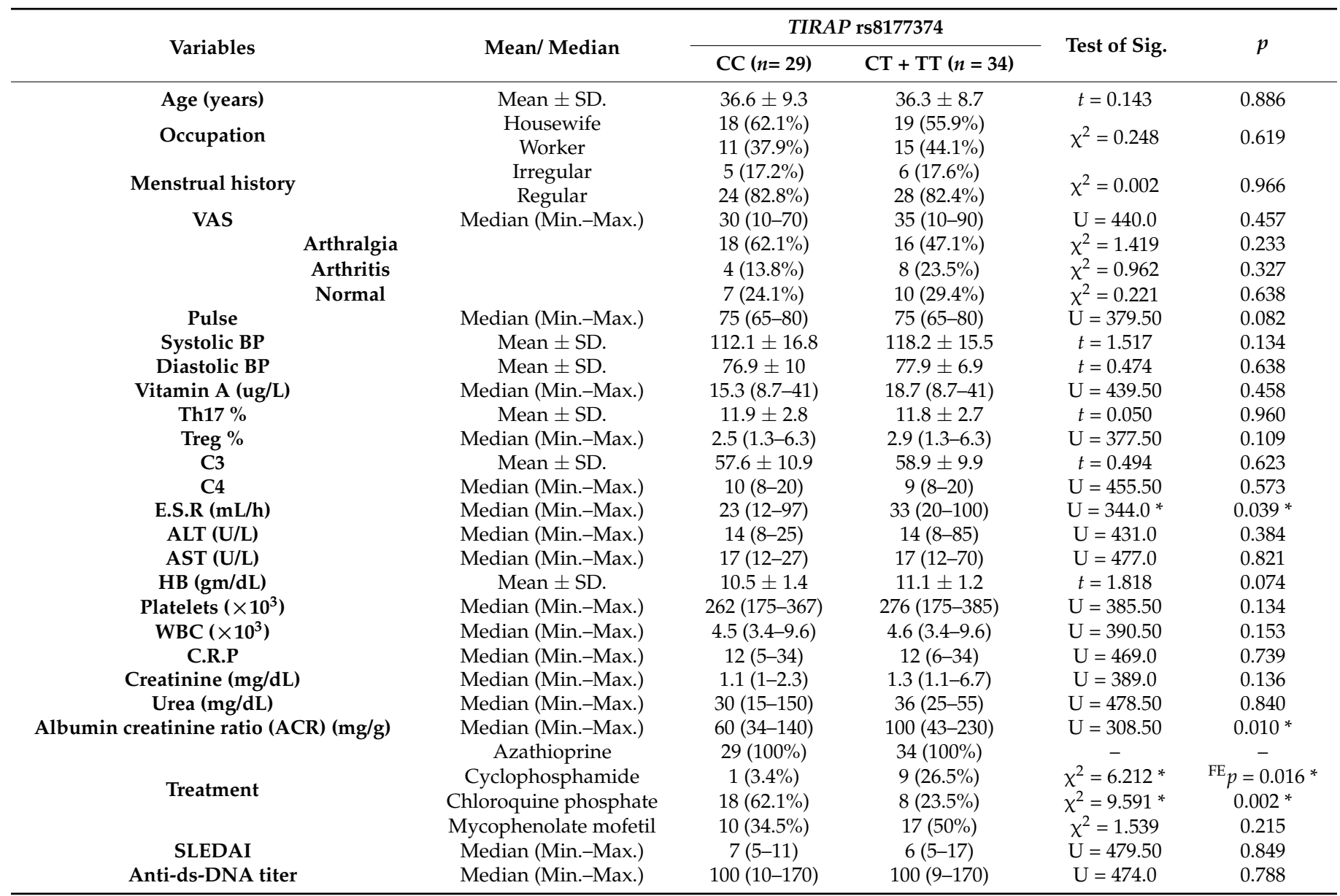

$\chi^{2}$ : chi-squared test; FE: Fisher's exact test; $t$ : Student's $t$-test; U: Mann-Whitney test. $p$ : $p$-value for comparing between the studied groups.

*: Statistically significant at $p \leq 0.05$. 
a
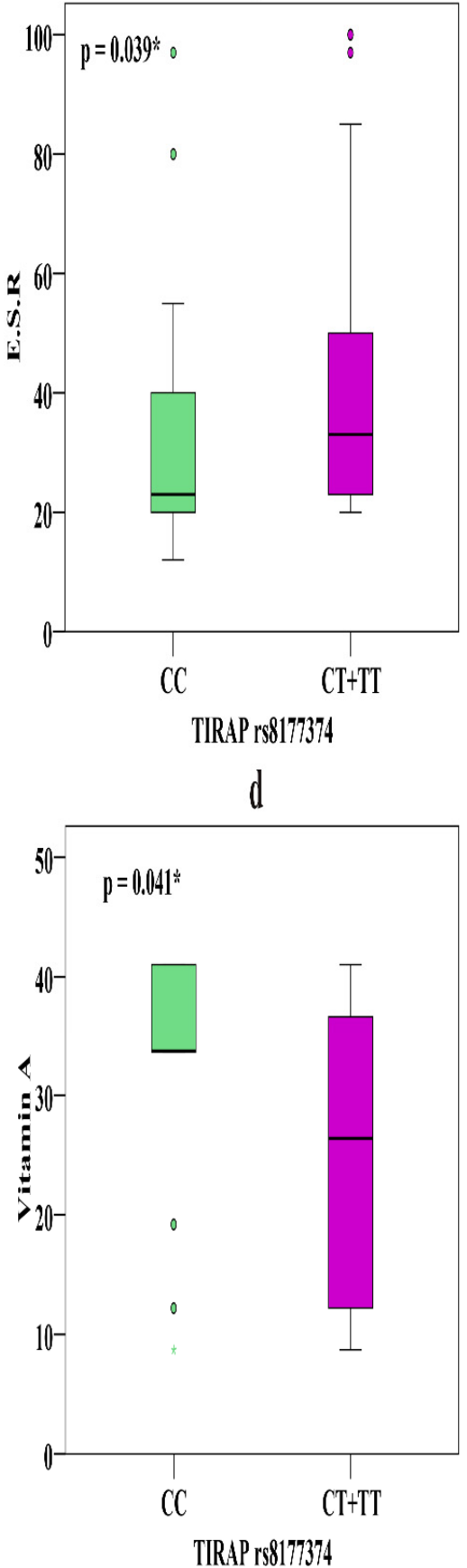
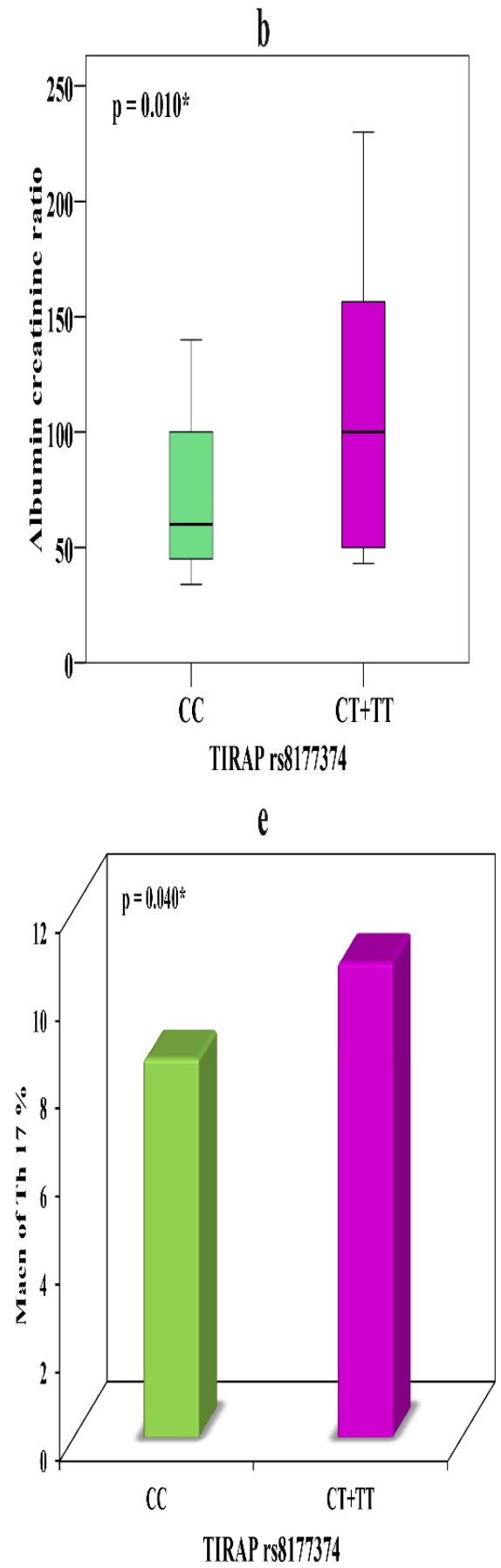
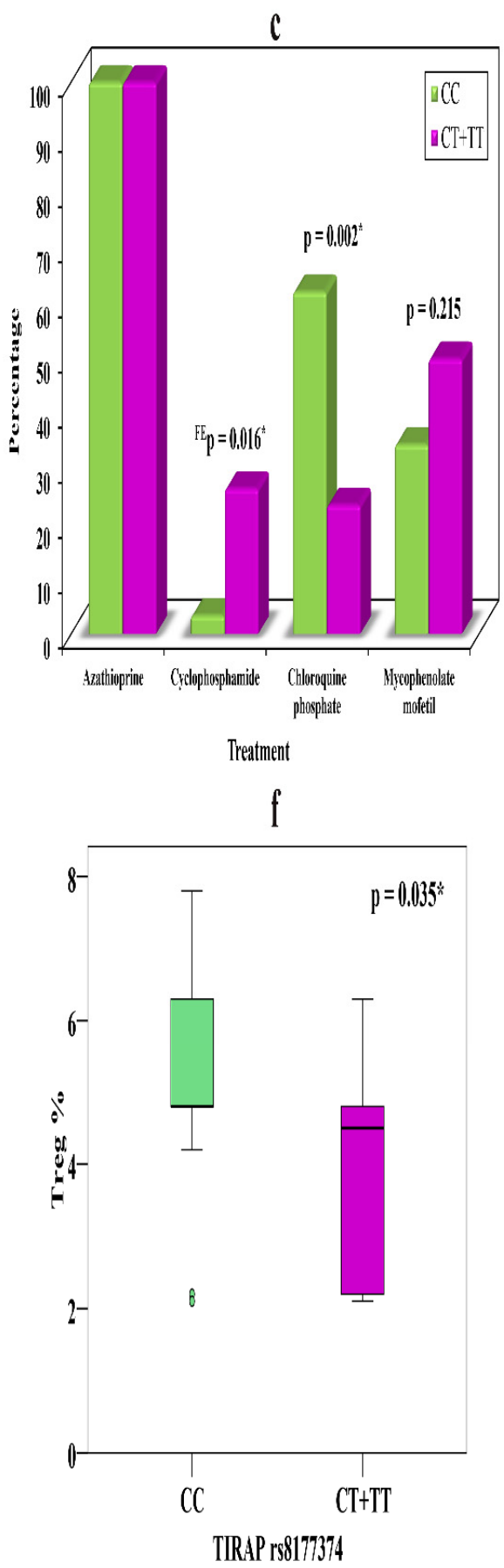

Figure 2. (a) Relationship between TIRAP rs8177374 and ESR in LN. (b) Relationship between TIRAP rs8177374 and ACR in LN. (c) Relationship between TIRAP rs8177374 and treatment in LN. (d) Relationship between TIRAP rs8177374 and vitamin A in patients without nephritis. (e) Relationship between TIRAP rs 8177374 and Th17 \% in patients without nephritis. (f) Relationship between TIRAP rs8177374 and Treg \% in patients without nephritis. *_significant.

Following adjustment for age, SBP, DBP, and arthralgia and arthritis patterns, univariate, and multivariate regression analysis of all SLE patients relative to controls revealed that the MECP2 rs2734647 genotypes CT and TT could be independent predictor of disease, with (OR 2.951, $p=0.001)$ and (OR 4.343, $p=0.015)$, respectively.

Moreover, TIRAP rs 8177374 genotypes CT and TT could also predict the disease, with (OR 3.274, $p<0.001$ ) and (OR 6.540, $p=0.005)$, respectively.

Univariate and multivariate regression analysis for SLE patients with and without nephritis revealed that the MECP2 rs2734647 genotypes CT and TT are independent 
predictors of nephritis in SLE patients, with (OR 2.70, $p=0.016)$ and (OR 2.648, $p=0.034$ ), respectively.

Moreover, the TIRAP rs8177374 genotypes CT and TT could also predict nephritis in SLE, with (OR 3.420, $p=0.003$ ) and (OR 3.942, $p=0.006)$, respectively.

\section{Discussion}

Systemic lupus erythematosus (SLE) is a clinicopathologically diverse chronic autoimmune disease that causes distress to various tissues and organs [23]. In Egypt, 89.7\% of SLE patients are females, while only $10.3 \%$ are males. The mutual cumulative displays include arthritis, malar rash, leukopenia, photosensitivity, nephritis, and neuropsychiatric lupus [24].

Genetic, epigenetic, and environmental factors might play crucial roles in the advance of SLE [25]. Recurrent flares and sustained phases of active illness are concomitant with adverse outcomes in lupus patients, and cause damage to tissues and organs [26]. LN is a dangerous outcome of SLE, adding considerably to SLE-related morbidity and mortality [27]. Early recognition and management of LN are imperative to diminish the risk of inflammation-prompted permanent kidney damage, and to reserve renal function. Additionally, the study of pathway-specific immune deregulation can enable accurate, personalized pharmacotherapy for LN [28].

We aimed in the present study to evaluate the connection between the MECP2 rs2734647 and TIRAP rs8177374 variants and SLE risk, and to establish their relationships with immune markers and medical management, in addition to disease progression and activity. Moreover, we decided to resolve their relationship with LN and their impact on kidney functions. We found that the T allele of $M E C P 2$ rs2734647 increases the risk of SLE. Additionally, we noticed that the T allele of TIRAP rs 8177374 also increases disease risk. These striking findings may prove both genes' association with SLE susceptibility. $\mathrm{MECP} 2$ recruits the histone deacetylase to the promoter regions of goal genes that provoke heterochromatin construction and transcriptional embarrassment [29]; additionally, it can control gene expression via DNMT1 recruitment. MECP2 reduces the release of IFN- $\gamma$ by Th cells, producing a partial immune inhibition [30]. Previous studies revealed a possible genetic association of $M E C P 2$ single-nucleotide polymorphisms (SNPs) with susceptibility to SLE [23,29,31-34]; moreover, MECP2 rs2734647 is related to other diseases, such as Rett syndrome [35], schizophrenia [36], and aggressive social behavior [37].

$M E C P 2$ is reflected as a gene related to SLE, attributed to its crucial function in the transcriptional suppression of genes that elude methylation and are highly expressed in SLE, and owing to the distorted control of T-cell genes, which are sensitive to methylation $[14,29,38]$.

Doudar et al. [31] found that the A allele of MECP2 rs1734791 is the risk allele for lupus in Egyptian populations. Rzeszotarska et al. [34] stated that rs2075596, rs1734787, rs17435, and rs2239464 within the MECP2 gene are more common in SLE patients than in healthy controls and could possibly constitute predictive elements for the progression and course of SLE in Polish populations. Alesaeidi et al. [39] found that in Iranian patients, rs1734787 and rs1734791 of $M E C P 2$ were correlated with SLE progression.

Studies on the relationship of TIRAP rs8177374 (C/T) polymorphism with SLE are limited; thus, we found a remarkable association with SLE susceptibility. TIRAP rs8177374, which codes a leucine replacement at serine 180 of Mal (S180L), was found to shield against pneumococcal disease, bacteremia, malaria, tuberculosis, and SLE [6,40]. Rupasree et al. [41] declared that the TLR4, TLR9, and TIRAP polymorphisms have an association with serological and phenotypic subsets of SLE via changes in MHC2TA and HLA-DR expression. Loss of TIRAP expression in SLE creates debate on the variants of significance in many populations, as is the case for many immunogenetic variants [5].

Few studies have inspected the relationship between MECP2 and TIRAP polymorphisms and LN, so we intended to examine their correlation with nephritis. We found that the $\mathrm{T}$ alleles of MECP2 rs2734647 and TIRAP rs8177374 were considerably higher in 
LN patients than in non-nephritis patients and controls; however, the $\mathrm{T}$ allele difference between non-nephritis patients and controls was not significant. Concerning the relationships of the studied SNPs with clinical features of SLE, CT + TT of MECP2 rs2734647 was correlated with elevated ACR, higher SLEDAI, and therapeutic drugs, while CT + TT of TIRAP rs8177374 was correlated with elevated ACR, higher ESR, and therapeutic drugs in nephritis patients, which may contribute to disease severity and progression, and could be linked with SLE prognosis. In non-nephritis patients, CT + TT of MECP2 was associated with lower vitamin A and elevated ACR and showed a better response to chloroquine phosphate than CC carriers, yet CT + TT of TIRAP showed noticeably lower vitamin A and Treg $\%$ and elevated Th17 \% relative to CC carriers.

Bentham et al. [42] announced in their GWAS analysis that MECP2 rs1734787 is an SLE risk factor for people of European ancestry. Rzeszotarska et al. [34] found that variants within the MECP2 gene (rs1734787 and rs2239464) might be related to earlier disease inception and more rapid disease passage and concluded that variants in the MECP2 and CCR5 genes might have an impact on elevated ALT and AST values. Conversely, Doudar et al. [31] did not detect a significant relationship between rs1734791 in MECP2 and disease severity or activity. Additionally, Alesaeidi et al. [39] demonstrated no significant connection between rs1734791 or rs1734787 in MECP2 and clinical features of SLE.

Rupasree et al. [41] declared that TIRAP S180L showed a positive association with alopecia and malar rashes, and an inverse relation with psychosis. TLR T399I and TIRAP S180L showed positive associations with anti-Ro, demonstrating the influence of TLR and TIRAP genotypes on specific autoantibody formation. Endogenous nuclear particles experiencing receptor-mediated endocytosis can extend endosomes and interact with endosomal TLRs. Genetic variants of TLR3 (dsRNA), TLR7 / 8 (ssRNA), and TLR9 (DNA) can precipitate LN. Activation of TLR3 on antigen-presenting cells (APCs) or renal mesangial cells can aggravate LN by enhancing the expression of CXCL1/GRO $\alpha$ to recruit PMNs to the inflammation area, contributing to renal damage [43,44].

Autoantibodies to double-stranded DNA (dsDNA), and markers of complement activation such as $\mathrm{C} 3$ and $\mathrm{C} 4$, are widely utilized in clinical practice in the diagnosis and surveillance of patients with LN, and these LN biomarkers were evidently used in this study. TIRAP is implicated in the TLR2- and TLR4-mediated MyD88-dependent signaling pathways; thus, TIRAP gene variants can modulate the signaling pathways and further the secretion of cytokines and inflammatory mediators, which can influence the course and progression of SLE. Chloroquine phosphate causes endosomal TLR blockade, which modulates the inflammatory process and cytokine production, but because of its serious side effects, the dose that we used in the clinic is not effective in all cases [45]. A previous animal study postulated that the administration of mycophenolate mofetil could inhibit TLR4 expression and signaling on the surface of monocytes, thus hindering the monocytes' immune function. Therefore, the immune markers were downregulated with the administration of mycophenolate mofetil, due to inhibition of cytokines and overproduction of chemokines, and could protect the renal tissues [46]. Cyclophosphamide is an alkylating agent that is used in severe cases of LN to suppress the immune system by diminishing the overproduction and infiltration of neutrophils [47], as well as downregulation of TLR2, which activates cytokines such as IL-10 by differentiating the Treg cells (TLR2-expressing dependent cell), which are the most important sources of IL-10 [48].

Many known LN susceptibility genes have functions that mediate inflammation via cytokine production and activation of leukocytes. The known cellular pathways mediated by these variant gene products also provide valuable mechanistic insights for the development of personalized therapeutics. Defining genetic variants that reliably predict disease susceptibility, risk of progression, and response to treatment carries tremendous potential to improve and personalize patient care [49].

Finally, a genome-wide association (GWA) study in Koreans revealed 10 risk regionsSTAT1-STAT4, TNFSF4, TNFAIP3, IKZF1, HIP1, IRF5, BLK, WDFY4, ETS1, and IRAK1MECP2- to be implicated in SLE [50]. 
The limitations of this study include its small sample size; thus, we recommend a wide-scale study in multiple centers to validate our results. If proved, these genetic tests could be used in the routine lab workup of SLE patients to predict their risk of LN, and whether or not they need extensive follow-up. Moreover, it would be better to conduct a long-term follow-up study to investigate the possible correlation between outcomes of specific treatments and drugs used, to validate the use of personalized medicine in SLE. Furthermore, we encourage further studies to establish correlations between $M E C P$ and TIRAP variants, with their relative gene expression levels, in order to investigate possible relationships with SLE pathogenesis and outcomes.

\section{Conclusions}

The genetic loci within the MECP2 and TIRAP genes could play a role in predisposition to SLE and can also predict disease onset and severity. The association of TIRAP polymorphism with lack of Treg percentage, decline in vitamin A, and rise in Th17percentage can facilitate SLE pathology. Moreover, the association of MECP2 and TIRAP with ACR and SELDAI can influence SLE activity and progress to lupus nephritis. We recommend further investigations regarding TLRs and their signaling pathways in LN that might identify a therapeutic target and permit accurate personalized therapy in LN.

Supplementary Materials: The following are available online at https:/ /www.mdpi.com/article/10 .3390 / biom11091378/s1, Figure S1: Figure S1 Allele discrimination plots and sequencing analysis were constructed for MECP2 and TIRAP.

Author Contributions: All authors contributed to the study's conception and design. Material preparation, data collection, and analysis were performed by S.I.T., S.E.S., D.S.F., N.M.M. and H.S.E. The first draft of the manuscript was written by S.I.T., S.E.S., E.A.E.-M., H.I.A.-E. and A.E.T., and all authors commented on previous versions of the manuscript. All authors have read and agreed to the published version of the manuscript.

Funding: This research received no external funding.

Institutional Review Board Statement: The study was conducted according to the guidelines of the Declaration of Helsinki and approved by the Research Ethics Committee of Faculty of Medicine, Menoufia University.

Informed Consent Statement: Informed consent was obtained from all subjects involved in the study.

Data Availability Statement: All data and materials support published claims and comply with field standards. The datasets generated and/or analyzed during the current study are not publicly available but are available from the corresponding author on reasonable request.

Conflicts of Interest: The authors declare no conflict of interest.

\section{References}

1. Dörner, T.; Furie, R. Novel paradigms in systemic lupus erythematosus. Lancet 2019, 393, 2344-2358. [CrossRef]

2. Mok, C.C. Therapeutic options for resistant lupus nephritis. Semin. Arthritis Rheum. 2006, 36, 71-81. [CrossRef] [PubMed]

3. Zhang, Y.; Liu, J.; Wang, C.; Liu, J.; Lu, W. Toll-Like Receptors Gene Polymorphisms in Autoimmune Disease. Front. Immunol. 2021, 12, 672346. [CrossRef]

4. Rajpoot, S.; Wary, K.K.; Ibbott, R.; Liu, D.; Saqib, U.; Thurston, T.L.M.; Baig, M.S. TIRAP in the Mechanism of Inflammation. Front. Immunol. 2021, 12, 697588. [CrossRef]

5. Belhaouane, I.; Hoffmann, E.; Chamaillard, M.; Brodin, P.; Machelart, A. Paradoxical Roles of the MAL/Tirap Adaptor in Pathologies. Front. Immunol. 2020, 11, 569127. [CrossRef] [PubMed]

6. Castiblanco, J.; Varela, D.C.; Castano-Rodriguez, N.; Rojas-Villarraga, A.; Hincapie, M.E.; Anaya, J.M. TIRAP (MAL) S180L polymorphism is a common protective factor against developing tuberculosis and systemic lupus erythematosus. Infect. Genet. Evol. 2008, 8, 541-544. [CrossRef]

7. Ferwerda, B.; Alonso, S.; Banahan, K.; McCall, M.B.; Giamarellos-Bourboulis, E.J.; Ramakers, B.P.; Mouktaroudi, M.; Fain, P.R.; Izagirre, N.; Syafruddin, D.; et al. Functional and genetic evidence that the Mal/TIRAP allele variant 180L has been selected by providing protection against septic shock. Proc. Natl. Acad. Sci. USA 2009, 106, 10272-10277. [CrossRef] [PubMed] 
8. Keeling, S.O.; Bissonauth, A.; Bernatsky, S.; VanderMeer, B.; Fortin, P.R.; Gladman, D.D.; Peschken, C.; Urowitz, M.B. Practice Variations in the Diagnosis, Monitoring, and Treatment of Systemic Lupus Erythematosus in Canada. J. Rheumatol. 2018, 45, 1440-1447. [CrossRef] [PubMed]

9. Hedrich, C.M.; Crispin, J.; Tsokos, G.C. Epigenetic regulation of cytokine expression in systemic lupus erythematosus with special focus on T cells. Autoimmunity 2014, 47, 234-241. [CrossRef]

10. Hedrich, C.M.; Mabert, K.; Rauen, T.; Tsokos, G.C. DNA methylation in systemic lupus erythematosus. Epigenomics 2017, 9, 505-525. [CrossRef]

11. Koelsch, K.A.; Webb, R.; Jeffries, M.; Dozmorov, M.G.; Frank, M.B.; Guthridge, J.M.; James, J.A.; Wren, J.D.; Sawalha, A.H. Functional characterization of the MECP2/IRAK1 lupus risk haplotype in human T cells and a human MECP2 transgenic mouse. J. Autoimmun. 2013, 41, 168-174. [CrossRef] [PubMed]

12. Huang, Y.-H.; Li, S.-C.; Huang, L.-H.; Chen, P.-C.; Lin, Y.-Y.; Lin, C.-C.; Kuo, H.-C. Identifying genetic hypomethylation and upregulation of Toll-like receptors in Kawasaki disease. Oncotarget 2017, 8, 11249-11258. [CrossRef]

13. Maslinska, D.; Laure-Kamionowska, M.; Maslinski, S. Original article. Methyl-CpG binding protein 2, receptors of innate immunity and receptor for advanced glycation end-products in human viral meningoencephalitis. Folia Neuropathol. 2014, 4, 428-435. [CrossRef]

14. Webb, R.; Wren, J.D.; Je_ries, M.A.; Kelly, J.A.; Kaufman, K.M.; Tang, Y.; Frank, M.B.; Merrill, J.T.; Kimberly, R.; Edberg, J.C.; et al. Variants within MECP2, a key transcription regulator, are associated with increased susceptibility to lupus and di_erential gene expression in patients with systemic lupus erythematosus. Arthritis Rheum. 2009, 60, 1076-1084. [CrossRef] [PubMed]

15. Aringer, M.; Costenbader, K.; Daikh, D.; Brinks, R.; Mosca, M.; Ramsey-Goldman, R.; Smolen, J.S.; Wofsy, D.; Boumpas, D.T.; Kamen, D.L.; et al. 2019 European League Against Rheumatism/American College of Rheumatology classification criteria for systemic lupus erythematosus. Ann. Rheum Dis. 2019, 78, 1151-1159. [CrossRef] [PubMed]

16. Aringer, M.; Costenbader, K.; Daikh, D.; Brinks, R.; Mosca, M.; Ramsey-Goldman, R.; Smolen, J.S.; Wofsy, D.; Boumpas, D.T.; Kamen, D.L.; et al. 2019 European League Against Rheumatism/American College of Rheumatology Classification Criteria for Systemic Lupus Erythematosus. Arthritis Rheumatol. 2019, 71, 1400-1412. [CrossRef]

17. Bombardier, C.; Gladman, D.D.; Urowitz, M.B.; Caron, D.; Chang, C.H. Derivation of the SLEDAI. A disease activity index for lupus patients. The Committee on Prognosis Studies in SLE. Arthritis Rheum. 1992, 35, 630-640. [CrossRef] [PubMed]

18. Hahn, B.H.; McMahon, M.A.; Wilkinson, A.; Wallace, W.D.; Daikh, D.I.; FitzGerald, J.; Karpouzas, G.A.; Merrill, J.T.; Wallace, D.J.; Yazdany, J.; et al. American College of Rheumatology guidelines for screening, treatment, and management of lupus nephritis. Arthritis Care Res. 2012, 64, 797-808. [CrossRef] [PubMed]

19. Izmirly, P.M.; Wan, I.; Sahl, S.; Buyon, J.; Belmont, H.; Salmon, J.E.; Askanase, A.; Bathon, J.M.; Geraldino-Pardilla, L.; Ali, Y.; et al. The incidence and prevalence of systemic lupus erythematosus in New York County (Manhattan), New York: The Manhattan Lupus Surveillance Program. Arthritis Rheumatol. 2017, 69, 2006-2017. [CrossRef]

20. Dall'Era, M.; Cisternas, M.G.; Snipes, K.; Herrinton, L.J.; Gordon, C.; Helmick, C.G. The incidence and prevalence of systemic lupus erythematosus in San Francisco County, California: The California Lupus Surveillance Project. Arthritis Rheumatol. 2017, 69, 1996-2005. [CrossRef] [PubMed]

21. Somers, E.C.; Marder, W.; Cagnoli, P.; Lewis, E.E.; Deguire, P.; Gordon, C.; Helmick, C.G.; Wang, L.; Wing, J.J.; Dhar, J.P.; et al. Population-based incidence and prevalence of systemic lupus erythematosus: The Michigan Lupus Epidemiology and Surveillance program. Arthritis Rheumatol. 2014, 66, 369-378. [CrossRef]

22. Crichton, N.; Wewers, M.E.; Lowe, N.K.; Liang, S.; Li, J.; Napierata, L. A critical review of visual Analogue scales in the measurement of clinical phenomena. J. Clin. Nurs. 2001, 10, 697-706.

23. Gachpazan, M.; Akhlaghipour, I.; Rahimi, H.R.; Saburi, E.; Mojarrad, M.; Abbaszadegan, M.R.; Moghbeli, M. Genetic and molecular biology of systemic lupus erythematosus among Iranian patients: An overview. Auto Immun. Highlights 2021, 12, 2, PMCID:PMC7847600. [CrossRef] [PubMed]

24. El Hadidi, K.T.; Medhat, B.M.; Baki, N.M.A.; Kafy, H.A.; Abdelrahaman, W.; Yousri, A.Y.; Attia, D.H.; Eissa, M.; El Dessouki, D.; Elgazzar, I.; et al. Characteristics of systemic lupus erythematosus in a sample of the Egyptian population: A retrospective cohort of 1109 patients from a single center. Lupus 2018, 27, 1030-1038. [CrossRef] [PubMed]

25. Tsokos, G.C. Autoimmunity and organ damage in systemic lupus erythematosus. Nat. Immunol. 2020, 21, 605-614. [CrossRef] [PubMed]

26. Alarcón, G.; Ugarte-Gil, M.; Pons-Estel, G.; Vilá, L.; Reveille, J.; McGwin, G., Jr. Remission, and low disease activity state (LDAS) are protective of intermediate and long-term outcomes in SLE patients. Results from LUMINA (LXXVIII), a multiethnic, multicenter US cohort. Lupus 2019, 28, 423-426. [CrossRef] [PubMed]

27. Borchers, A.T.; Leibushor, N.; Naguwa, S.M.; Cheema, G.S.; Shoenfeld, Y.; Gershwin, M.E. Lupus nephritis: A critical review. Autoimmun. Rev. 2012, 12, 174-194. [CrossRef] [PubMed]

28. Rullo, O.J.; Tsao, B.P. Recent insights into the genetic basis of systemic lupus erythematosus. Ann. Rheum. Dis. 2012, 72 (Suppl. 2), ii56-ii61. [CrossRef]

29. Sawalha, A.H.; Webb, R.; Han, S.; Kelly, J.A.; Kaufman, K.M.; Kimberly, R.P.; Alarcon-Riquelme, M.E.; James, J.A.; Vyse, T.J.; Gilkeson, G.S.; et al. Common variants within MECP2 confer risk of systemic lupus erythematosus. PLoS ONE 2008, 3, e1727, PMCID:PMC2253825. [CrossRef] [PubMed] 
30. Yang, T.; Ramocki, M.B.; Neul, J.L.; Lu, W.; Roberts, L.; Knight, J.; Ward, C.S.; Zoghbi, H.Y.; Kheradmand, F.; Corry, D.B. Overexpression of methyl-CpG binding protein 2 impairs T(H)1 responses. Sci. Transl. Med. 2012, 4, 163ra158, PMCID:PMC3628825. [CrossRef] [PubMed]

31. Doudar, N.A.; Abdelshafy, S.S.; Rady, S.A.; Mokhtar, A.M. Systemic lupus erythematosus: Genetic variants in Xq28 region. Reumatologia 2019, 57, 264-270, PMCID:PMC6911245. [CrossRef] [PubMed]

32. Kaufman, K.M.; Zhao, J.; Kelly, J.A.; Hughes, T.; Adler, A.; Sanchez, E.; Ojwang, J.O.; Langefeld, C.D.; Ziegler, J.T.; Williams, A.H.; et al. Fine mapping of Xq28: Both MECP2 and IRAK1 contribute to risk for systemic lupus erythematosus in multiple ancestral groups. Ann. Rheum. Dis. 2013, 72, 437-444, PMCID:PMC3567234. [CrossRef] [PubMed]

33. Uzrail, A.H.; Assaf, A.M.; Abdalla, S.S. Correlations of Expression Levels of a Panel of Genes (IRF5, STAT4, TNFSF4, MECP2, and TLR7) and Cytokine Levels (IL-2, IL-6, IL-10, IL-12, IFN- $\gamma$, and TNF- $\alpha$ ) with Systemic Lupus Erythematosus Outcomes in Jordanian Patients. BioMed Res. Int. 2019, 2019, 1703842, PMCID:PMC6907047. [CrossRef] [PubMed]

34. Rzeszotarska, E.; Sowinska, A.; Stypinska, B.; Walczuk, E.; Wajda, A.; Lutkowska, A.; Felis-Giemza, A.; Olesinska, M.; Puszczewicz, M.; Majewski, D.; et al. The Role of MECP2 and CCR5 Polymorphisms on the Development and Course of Systemic Lupus Erythematosus. Biomolecules 2020, 10, 494, PMCID:PMC7175371. [CrossRef] [PubMed]

35. Smeets, E.E.; Pelc, K.; Dan, B. Rett Syndrome. Mol. Syndr. 2011, 2, 113-127, PMCID:PMC3366703. [CrossRef] [PubMed]

36. Wong, E.H.; So, H.-C.; Li, M.; Wang, Q.; Butler, A.W.; Paul, B.; Wu, H.-M.; Hui, T.C.; Choi, S.-C.; So, M.-T.; et al. Common variants on Xq28 conferring risk of schizophrenia in Han Chinese. Schizophr. Bull. 2013, 40, 777-786, PMCID:PMC4059435. [CrossRef] [PubMed]

37. Tantra, M.; Hammer, C.; Kästner, A.; Dahm, L.; Begemann, M.; Bodda, C.; Hammerschmidt, K.; Giegling, I.; Stepniak, B.; Venzor, A.C.; et al. Mild expression differences of MECP2 influencing aggressive social behavior. EMBO Mol. Med. 2014, 6, 662-684, PMCID:PMC4023888. [CrossRef] [PubMed]

38. Suarez-Gestal, M.; Calaza, M.; Endreffy, E.; Pullmann, R.; Ordi-Ros, J.; Sebastiani, G.D.; Ruzickova, S.; Santos, M.J.; Papasteriades, C.; Marchini, M.; et al. Replication of recently identified systemic lupus erythematosus genetic associations: A case-control study. Arthritis Res. Ther. 2009, 11, R69, PMCID:PMC2714115. [CrossRef] [PubMed]

39. AleSaeidi, S.; Karami, J.; Mahmoudi, M.; Akbarian, M.; Poursani, S.; Amirzadeh, A.; Haddadi, N.-S.; Saffari, E.; Jamshidi, A.R. Methyl-CpG-Binding Protein 2 (MECP2) Polymorphism in Iranian Patients with Systemic Lupus Erythematosus. Inflammation 2015, 38, 2185-2190. [CrossRef] [PubMed]

40. Khor, C.C.; Chapman, S.J.; Vannberg, F.O.; Dunne, A.; Murphy, C.; Ling, E.Y.; Frodsham, A.J.; Walley, A.J.; Kyrieleis, O.; Khan, A.; et al. A Mal functional variant is associated with protection against invasive pneumococcal disease, bacteremia, malaria, and tuberculosis. Nat. Genet. 2007, 39, 523-528, PMCID:PMC2660299. [CrossRef] [PubMed]

41. Rupasree, Y.; Naushad, S.M.; Rajasekhar, L.; Uma, A.; Kutala, V.K. Association of TLR4 (D299G, T399I), TLR9-1486T>C, TIRAP S180L and TNF- $\alpha$ promoter $(-1031,-863,-857)$ polymorphisms with risk for systemic lupus erythematosus among South Indians. Lupus 2015, 24, 50-57. [CrossRef] [PubMed]

42. Bentham, J.; Morris, D.; Graham, D.S.C.; Pinder, C.; Tombleson, P.; Behrens, T.W.; Martin, J.; Fairfax, B.P.; Knight, J.; Chen, L.; et al. Genetic association analyses implicate aberrant regulation of innate and adaptive immunity genes in the pathogenesis of systemic lupus erythematosus. Nat. Genet. 2015, 47, 1457-1464, PMCID:PMC4668589. [CrossRef] [PubMed]

43. Enevold, C.; Kjær, L.; Nielsen, C.H.; Voss, A.; Jacobsen, R.S.; Hermansen, M.L.F.; Redder, L.; Oturai, A.B.; Jensen, P.E.; Bendtzen, K.; et al. Genetic polymorphisms of dsRNA ligating pattern recognition receptors TLR3, MDA5, and RIG-I. Association with systemic lupus erythematosus and clinical phenotypes. Rheumatol. Int. 2014, 34, 1401-1408. [CrossRef] [PubMed]

44. Imaizumi, T.; Aizawa, T.; Segawa, C.; Shimada, M.; Tsuruga, K.; Kawaguchi, S.; Matsumiya, T.; Yoshida, H.; Joh, K.; Tanaka, H. Toll-like receptor 3 signaling contributes to the expression of a neutrophil chemoattractant, CXCL1 in human mesangial cells. Clin. Exp. Nephrol. 2014, 19, 761-770. [CrossRef] [PubMed]

45. Suarez-Almazor, M.E.; Belseck, E.; Shea, B.; Homik, J.; Wells, G.A.; Tugwell, P. Antimalarials for treating rheumatoid arthritis. Cochrane Database Syst. Rev. 2000, 4, CD000959. [CrossRef] [PubMed]

46. Zhang, Y.X.; Zhang, J.R.; Wang, Z.G. Mycophenolate mofetil affects monocyte Toll-like receptor 4 signaling during mouse renal ischemia/reperfusion injury. Chin. Med. J. 2013, 126, 1224-1229. [PubMed]

47. Nussbaumer, S.; Bonnabry, P.; Veuthey, J.-L.; Fleury-Souverain, S. Analysis of anticancer drugs: A review. Talanta 2011, 85, 2265-2289. [CrossRef] [PubMed]

48. Andrade, E.B.; Alves, J.; Madureira, P.; Oliveira, L.; Ribeiro, A.; Cordeiro-Da-Silva, A.; Correia-Neves, M.; Trieu-Cuot, P.; Ferreira, P. TLR2-induced IL-10 production impairs neutrophil recruitment to infected tissues during neonatal bacterial sepsis. J. Immunol. 2013, 191, 4759-4768. [CrossRef]

49. Caster, D.J.; Powell, D.W. Utilization of Biomarkers in Lupus Nephritis. Adv. Chronic Kidney Dis. 2019, 26, 351-359, PMCID:PMC7001747. [CrossRef] [PubMed]

50. Lessard, C.J.; Sajuthi, S.; Zhao, J.; Kim, K.; Ice, J.A.; Li, H.; Ainsworth, H.; Rasmussen, A.; Kelly, J.; Marion, M.; et al. Identification of a Systemic Lupus Erythematosus Risk Locus Spanning ATG16L2, FCHSD2, and P2RY2 in Koreans. Arthritis Rheumatol. 2016, 68, 1197-1209. [CrossRef] [PubMed] 